\title{
POLARIZATIONS OF POWERS OF GRADED MAXIMAL IDEALS
}

\author{
AYAH ALMOUSA, GUNNAR FLØYSTAD, AND HENNING LOHNE
}

\begin{abstract}
We give a complete combinatorial characterization of all possible polarizations of powers of the graded maximal ideal $\left(x_{1}, x_{2}, \ldots, x_{m}\right)^{n}$ of a polynomial ring in $m$ variables. We also give a combinatorial description of the Alexander duals of such polarizations. In the three variable case $m=3$ and also in the power two case $n=2$ the descriptions are easily visualized and we show that every polarization defines a (shellable) simplicial ball. We give conjectures relating to topological properties and to algebraic geometry, in particular that any polarization of an Artinian monomial ideal defines a simplicial ball.
\end{abstract}

\section{INTRODUCTION}

The notion of polarization of a monomial ideal has been a standard technique in algebraic geometry and combinatorial commutative algebra for many years. It gives a way to "separate" the powers of variables in a monomial ideal while keeping its homological properties. An early success was Hartshorne's proof the connectedness of the Hilbert scheme [16] using distractions, which are essentially a form of polarization. Polarization gives a way to make combinatorial objects from any monomial ideal: it gives a squarefree monomial ideal and thus a simplicial complex, from which one may obtain algebraic invariants such as graded Betti numbers in a combinatorial/topological way. Although many authors have considered and used polarizations in various forms, there has been no focused, systematic study of the variety of polarizations, and of the characterizing properties of such ideals. The purpose of this article is to undertake this task. As we shall see, this raises both interesting questions in itself, as well as connections to combinatorial topology and to algebraic geometry manifesting in quite general conjectures.

Before proceeding let us briefly give simple examples and explain the notion of polarization. Consider the ideal

$$
I=\left(x_{1}, x_{2}\right)^{2}=\left(x_{1}^{2}, x_{1} x_{2}, x_{2}^{2}\right) \subseteq k\left[x_{1}, x_{2}\right] .
$$

It polarizes to the ideal

$$
J=\left(x_{11} x_{12}, x_{11} x_{21}, x_{21} x_{22}\right) \subseteq k\left[x_{11}, x_{12}, x_{21}, x_{22}\right] .
$$

Date: November 10, 2020.

2010 Mathematics Subject Classification. Primary: 13F20,13F55; Secondary: 55U10,05E40.

Key words and phrases. polarization, maximal ideal power, Artinian monomial ideal, isotone map, Alexander dual, simplicial ball, polynomial ring.

AA was partially supported by the NSF GRFP under Grant No. DGE-1650441. 
The quotient ring $k\left[x_{1}, x_{2}\right] / I$ then comes from $k\left[x_{11}, x_{12}, x_{21}, x_{22}\right] / J$ by cutting down by a regular sequence of variable differences

$$
x_{11}-x_{12}, x_{21}-x_{22} .
$$

These two graded rings have the same homological properties, such as codimension and codepth, and the same graded Betti numbers.

In general, for a monomial ideal $I$ one gets the polarization $J$ by taking each minimal generator of $I$

$$
x_{1}^{a_{1}} x_{2}^{a_{2}} \cdots x_{m}^{a_{m}}
$$

and making a minimal generator

$$
\left(x_{11} x_{12} \cdots x_{1 a_{1}}\right) \cdot\left(x_{21} x_{22} \cdots x_{2 a_{2}}\right) \cdots\left(x_{m 1} \cdots x_{m a_{m}}\right)
$$

of $J$. We call this the standard polarization.

However, for a monomial ideal $I$ in $k\left[x_{1}, \ldots x_{m}\right]$, there may be many other ways to get a squarefree monomial ideal $J$ in a larger polynomial ring $k\left[x_{i j}\right]$ such that $k\left[x_{i}\right] / I$ comes from $k\left[x_{i j}\right] / J$ by dividing out by a regular sequence of variable differences $x_{i, j}-x_{i, j^{\prime}}$.

For instance, if $I$ is a strongly stable ideal, one has the "b-polarization", [23], [28] which from the ideal (1) constructs the ideal

$$
J^{b}=\left(x_{11} x_{12}, x_{11} x_{22}, x_{21} x_{22}\right) \subseteq k\left[x_{11}, x_{12}, x_{21}, x_{22}\right] .
$$

(In this special case the ideals $J$ and $J^{b}$ are isomorphic, but this is not so in general.) The b-polarization takes the minimal generator (2) and makes a minimal generator

$$
\left(x_{1,1} \cdots x_{1, a_{1}}\right) \cdot\left(x_{2, a_{1}+1} \cdots x_{2, a_{1}+a_{2}}\right) \cdots\left(x_{m, a_{1}+\cdots+a_{m-1}+1} \cdots x_{m, a_{1}+\cdots+a_{m}}\right)
$$

so the second index runs trough the integers from 1 to $a_{1}+\cdots+a_{m}$.

The notion of shifting operator is a form of polarization. It has been studied by several authors, 21] in the setting of squeezed spheres, and in [28. Recently letterplace ideals associated to poset ideals of $P$-partitions, [12] and [13] have provided large classes which are new polarizations of Artinian monomial ideals.

1.1. Polarizations of powers of the graded maximal ideal. We combinatorially describe all possible polarizations of powers of the graded maximal ideal

$$
I=\left(x_{1}, \ldots, x_{m}\right)^{n} \subseteq k\left[x_{1}, \ldots, x_{m}\right] .
$$

These maximal ideal powers are the ideals in $k\left[x_{1}, \ldots, x_{m}\right]$ with maximal symmetry. They are $G L(n)$-invariant. A polarization is somehow a way of breaking this symmetry, but still keeping the homological properties. Thus we classify all symmetry breaks of this ideal. (Let us also mention that powers of graded maximal ideals have been studied from another combinatorial perspective in [2], using discrete Morse theory to make cellular resolutions.)

Let $\Delta_{m}(n)$ be the lattice simplex with vertices given by all $\mathbf{a}=\left(a_{1}, \ldots, a_{m}\right) \in \mathbb{N}_{0}^{m}$ with $\sum_{i=1}^{m} a_{i}=n$. Its vertices are in one-one correspondence with minimal generators of $\left(x_{1}, \ldots, x_{m}\right)^{n}$. See Figure 1 for $\Delta_{3}(3)$. It is divided into smaller triangles, and of these triangles, three are pointing down; we refer to these as down-triangles. By going 


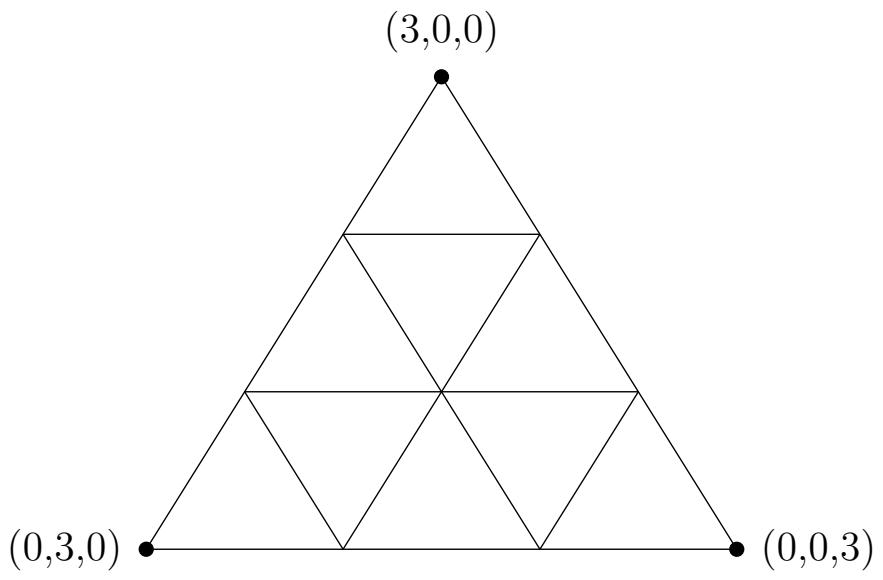

FiguRE 1.

upwards along the edges in this diagram, Figure 1, we get a partial order $\geq_{1}$ on $\Delta_{m}(n)$. If the monomial (2) polarizes to a monomial

$$
m(\mathbf{a})=m_{1}(\mathbf{a}) \cdots m_{m}(\mathbf{a})
$$

where $m_{i}(\mathbf{a})$ maps to $x_{i}^{a_{i}}$, let $X_{1}(\mathbf{a})$ be the variables in $m_{1}(\mathbf{a})$. Then $X_{1}$ can be considered as a map from $\Delta_{m}(n)$ to the boolean poset of subsets of the $x_{1 j}$-variables. Similarly, we get maps $X_{i}$ for each $i=1, \ldots, m$. For any polarization $J$ of $I$ in (3), it turns out that each $X_{i}$ is an isotone map for a partial order $\geq_{i}$ on $\Delta_{m}(n)$. Conversely, given the maps $\left\{X_{i}\right\}$, we can construct monomial generators $m(\mathbf{a})$ of an ideal $J$. When is this a polarization of $I$ ?

For a given edge in $\Delta_{m}(n)$ between $\mathbf{a}$ and $\mathbf{b}$, we call the edge a linear syzygy edge if there is a linear syzygy between the monomials $m(\mathbf{a})$ and $m(\mathbf{b})$. Our main result, Theorem 4.5, says that the maps $\left\{X_{i}\right\}$ determine a polarization of $I$ if and only if the linear syzygy edges for these maps contain a spanning tree of the edge graph (this is a complete graph) of each higher-dimensional "down-triangle" of $\Delta_{m}(n)$.

Two special cases are worth attention due to the easy visualization of the various polarizations. First, the polarizations of $\left(x_{1}, x_{2}, x_{3}\right)^{n}$, which are easily visualized in terms of Figure 1, see Corollary 4.7 and Example 4.8. Secondly, the polarizations of $\left(x_{1}, x_{2}, \ldots, x_{m}\right)^{2}$, which are in one-to-one correspondence with trees on $(m+1)$ vertices, Theorem 6.1 and Examples 6.2 and 6.3.

1.2. Polarizations of Artinian monomial ideals. A nice result on the topological properties of polarizations by S.Murai [22] is that the standard polarization of any Artinian monomial ideal gives a simplicial ball. The boundary is a simplicial sphere with a simply described Stanley-Reisner ideal, which generalizes Bier spheres, 5]. This construction has again been comprehensively generalized by A. D'Ali, A.Nematbakhsh and the second author in [7], by considering letterplace ideals.

We here give the full simple conjecture, Conjecture 3.2, that via the Stanley-Reisner correspondence, any polarization of an Artinian monomial ideal gives a simplicial ball with a simple and naturally described Stanley-Reisner ideal of its boundary, a simplicial 
sphere. We show that all polarizations of $\left(x_{1}, x_{2}, x_{3}\right)^{n}$ and of $\left(x_{1}, \ldots, x_{m}\right)^{2}$ give simplicial balls, by showing that the Alexander duals of all such polarizations have linear quotients. In a forthcoming paper we show the squeezed spheres of [18] and the generalized Bier spheres both fit into one and the same framework of polarization and deseparation which we give here, see Section 2 ,

Relating to algebraic geometry we conjecture, Conjecture 3.12, that every first order deformation of any polarization of any Artinian monomial ideal lifts to a global deformation. In particular, such a polarization would be a smooth point on any Hilbert scheme. If true, this would give a plethora of components of the Hilbert scheme even for the same Hilbert polynomial.

1.3. Alexander duals of polarizations. If $J$ is any polarization of an Artinian monomial ideal $I$ of $k\left[x_{1}, \ldots, x_{m}\right]$, then its Alexander dual $J^{\vee}$ will be generated by "colored" monomials of degree $m$, monomials of the form

$$
x_{1 j_{1}} x_{2 j_{2}} \cdots x_{m j_{m}}
$$

where for each $i=1, \ldots, m$, we have one variable $x_{i, j_{i}}$ from the class of $i$-variables (color $i$ ). We call these rainbow monomials. The class of ideals generated by rainbow monomials and with $m$-linear resolution is precisely the class which is Alexander dual to the class of polarizations of Artinian monomial ideals in $m$ variables, Proposition 3.6. A concise criterion for ideals generated by rainbow monomials to have linear resolution is given by A.Nematbakhsh in [24], and we recall it in Theorem 3.8.

We describe the Alexander dual of any polarization of the maximal ideal power $\left(x_{1}, \ldots, x_{m}\right)^{n}$, in terms of the isotone maps $X_{i}$. As it turns out, the argument involves only a baby-form $\chi_{i}$ of the isotone maps $X_{i}$, where $\chi_{i}$ is an isotone map from $\Delta_{m}(n)$ to the poset $\{0<1\}$. This description of the Alexander dual, however, leaves something to be desired concerning transparency; for instance, it is not obvious that the number of generators is actually always $\left(\begin{array}{c}n+m-1 \\ m\end{array}\right)$. We discuss problems concerning ideals generated by rainbow monomials in Section 3 .

1.4. Organization of the paper. In Section 2 we recall the notions of separations, separated models and polarizations. We show some basic results on polarizations of Artinian monomial ideals and introduce the isotone maps $X_{i}$. In Section 3 we discuss various conjectures and problems that have come up during our investigations. Section 4 contains our main result concerning the complete combinatorial classification of all polarizations of $\left(x_{1}, \ldots, x_{m}\right)^{n}$. Examples are given for the three-variable case. A short Section 5 classifies for the three-variable the isotone maps $\Delta_{3}(n) \rightarrow B(n)$. In Section 6 we consider the degree two case and show that polarizations of $\left(x_{1}, \ldots, x_{m}\right)^{2}$ are in oneto-one correspondence with trees on $(m+1)$ vertices. Section 7 gives the Alexander duals of polarizations of maximal ideal powers, and in Section 8 we show that polarizations of $\left(x_{1}, x_{2}, x_{3}\right)^{m}$ are shellable, which implies that they define simplicial balls by the Stanley-Reisner correspondence.

Note. The results in Section 6, Section 5, and Theorem 4.5 in the special case of three variables are essentially found in the unpublished preprint [20] by the third author. 


\section{Separations of MONOMial IDEALS}

We recall the basic notions of separation of a monomial ideals and separated models, as introduced in [13]. We also define a polarization of a monomial ideal as a separation which is a squarefree monomial ideal. We consider Artinian monomial ideals and show that for these the notion of polarization and separated models are the same. We introduce the isotone maps $X_{i}$ from the lattice simplex $\Delta_{m}(n)$ to the Boolean poset $B(n)$, which are our main gadgets to classify all the polarizations of maximal ideal powers.

If $R$ is a set, let $k\left[x_{R}\right]$ be the polynomial ring in the variables $x_{r}$ where $r \in R$. If $S \rightarrow R$ is a map of sets, it induces a $k$-algebra homomorphism $k\left[x_{S}\right] \rightarrow k\left[x_{R}\right]$ by mapping $x_{s}$ to $x_{r}$ if $s \mapsto r$.

\subsection{Separations and polarizations.}

Definition 2.1. Let $R^{\prime} \stackrel{p}{\longrightarrow} R$ be a surjection of finite sets with the cardinality of $R^{\prime}$ one more than that of $R$. Let $r_{1}$ and $r_{2}$ be the two distinct elements of $R^{\prime}$ which map to a single element $r$ in $R$. Let $I$ be a monomial ideal in the polynomial ring $k\left[x_{R}\right]$ and $J$ a monomial ideal in $k\left[x_{R^{\prime}}\right]$. We say $J$ is a simple separation of $I$ if the following holds:

i. The monomial ideal $I$ is the image of $J$ by the map $k\left[x_{R^{\prime}}\right] \rightarrow k\left[x_{R}\right]$.

ii. Both the variables $x_{r_{1}}$ and $x_{r_{2}}$ occur in some minimal generators of $J$ (usually in distinct generators).

iii. The variable difference $x_{r_{1}}-x_{r_{2}}$ is a non-zero divisor in the quotient ring $k\left[x_{R^{\prime}}\right] / J$.

More generally, if $R^{\prime} \stackrel{p}{\longrightarrow} R$ is a surjection of finite sets and $I \subseteq k\left[x_{R}\right]$ and $J \subseteq k\left[x_{R^{\prime}}\right]$ are monomial ideals such that $J$ is obtained by a succession of simple separations of $I$, $J$ is a separation of $I$. If $J$ has no further separation, we call $J$ a separated model (of $I)$.

A simple, but for us significant observation is that the minimal generators of the separation $J$ and the ideal $I$ are in one-one correspondence. More generally the graded Betti numbers of $J$ and $I$ are the same, since we get from $k\left[x_{R^{\prime}}\right] / J$ to $k\left[x_{R}\right] / I$ by dividing out by a regular sequence of linear forms.

In [1] it is shown that simple separations may be considered as deformations of the ideal $I$.

Any monomial ideal may be separated to its standard polarization. So clearly any separated model is a squarefree monomial ideal. The standard polarization may, however, be further separable, so it may not be a separated model.

Example 2.2. Consider $I=\left(x^{2} y^{2}, x^{2} z^{2}, y^{2} z^{2}\right)$ in $k[x, y, z]$. The standard polarization is

$$
\tilde{I}=\left(x_{1} x_{2} y_{1} y_{2}, x_{1} x_{2} z_{1} z_{2}, y_{1} y_{2} z_{1} z_{2}\right) \text {. }
$$

This may be further separated to

$$
J=\left(x_{1} x_{2} y_{1} y_{2}, x_{1}^{\prime} x_{2}^{\prime} z_{1} z_{2}, y_{1} y_{2} z_{1} z_{2}\right) .
$$


Definition 2.3. Let $I \subseteq k\left[x_{R}\right]$ be a monomial ideal and $R^{\prime} \rightarrow R$ be a surjection of finite sets. An ideal $J \subseteq k\left[x_{R^{\prime}}\right]$ is a polarization of $I$ if $J$ is squarefree and a separation of $I$.

This general notion of polarization is likely first defined in 28]. By the example above it is not true that any polarization is a separated model. However, we shall see in Corollary 2.7 that for Artinian monomial ideals, these notions are equivalent.

We state a general lemma which will be useful later.

Lemma 2.4. Let I be a monomial ideal in $k\left[x_{0}, x_{1}, \ldots, x_{m}\right]$ such that each generator of $I$ is squarefree in the $x_{0}$-variable. Then if $\left(x_{0}-x_{1}\right) \cdot f$ is in $I$, then for every monomial $m$ in $f$ we have that $x_{0} m$ and $x_{1} m$ are in $I$.

Proof. Let $f=x_{0}^{a} f_{a}+x_{0}^{a-1} f_{a-1}+\cdots+f_{0}$ where each $f_{p}$ have no $x_{0}$-terms. Then if $\left(x_{0}-x_{1}\right) f$ is in $I$, the only terms with $x_{0}^{a+1}$ are the terms in $x_{0}^{a+1} f_{a}$, and so these are in $I$ since we are in a $\mathbb{Z}^{m}$-graded setting. But since $I$ is squarefree in $x_{0}$, we have $x_{0} f_{a}$ in $I$ and so $x_{0}^{a} f_{a}$ in $I$. In this way we may peel off and get that all terms $x_{0}^{p} f_{p}$ are in $I$ for $p \geq 1$.

Then in $\left(x_{0}-x_{1}\right) f_{0}$, the terms with $x_{0}$ are those in $x_{0} f_{0}$. Hence $x_{0} f_{0}$ is in $I$ and so $x_{0} f$ is in $I$. Again since $I$ is multigraded, each monomial term $x_{0} m$ is in $I$. We also get $x_{1} f \in I$ and then each $x_{1} m \in I$.

2.2. Polarizations of Artinian monomial ideals. We consider an Artinian monomial ideal $I \subseteq k\left[x_{1}, \ldots, x_{m}\right]$. This is simply a monomial ideal such that for every index $i$, some power $x_{i}^{n_{i}}$ is a minimal generator of $I$. Let $\check{X}_{i}=\left\{x_{i 1}, x_{i 2}, \ldots, x_{i n_{i}^{\prime}}\right\}$ be a set of variables. We get a polynomial ring whose variables are those in the union of all these variables, and a homomorphism

$$
\pi: k\left[\check{X}_{1}, \check{X}_{2}, \ldots, \check{X}_{m}\right] \rightarrow k\left[x_{1}, \ldots, x_{m}\right]
$$

by mapping every variable in $\check{X}_{i}$ to $x_{i}$.

In a polarization $J \subseteq k\left[\check{X}_{1}, \ldots, \check{X}_{m}\right]$ of $I$, we separate each monomial generator $x^{\mathbf{a}}=x_{1}^{a_{1}} x_{2}^{a_{2}} \cdots x_{m}^{a_{m}}$ to squarefree monomials

$$
m(\mathbf{a})=m_{1}(\mathbf{a}) \cdot m_{2}(\mathbf{a}) \cdots m_{m}(\mathbf{a}),
$$

where $m_{i}(\mathbf{a})$ is a squarefree monomial of degree $a_{i}$ in variables from $\check{X}_{i}$. Considering $x_{i}^{n_{i}}$ we see that we must have $n_{i}^{\prime} \geq n_{i}$. We shall shortly show, Remark 2.6, that we may take $n_{i}^{\prime}=n_{i}$.

Starting from $K\left[\check{X}_{1}, \ldots, \check{X}_{m}\right] / J$ we get the quotient ring $k\left[x_{1}, \ldots, x_{m}\right] / I$ be dividing out by a regular sequence consisting of variable differences $x_{i p}-x_{i q}$. For each $i$ we choose $\left(n_{i}-1\right)$ linearly independent such variable differences. Any such sequence of variable differences in any order will do.

We may get intermediate separations of $I$ as follows. Choose surjections $p_{i}: \check{X}_{i} \rightarrow \check{X}_{i}^{\prime}$. We get a map of polynomial rings

$$
k\left[\check{X}_{1}, \ldots, \check{X}_{m}\right] \rightarrow k\left[\check{X}_{1}^{\prime}, \ldots, \check{X}_{m}^{\prime}\right] .
$$

The image of the polarization $J$ is an ideal $I^{\prime}$ in $k\left[\check{X}_{1}^{\prime}, \ldots, \check{X}_{m}^{\prime}\right]$ and $I^{\prime}$ is a separation of $I$. We then get from $k\left[\check{X}_{1}, \ldots, \check{X}_{m}\right] / J$ to $k\left[\check{X}_{1}^{\prime}, \ldots, \check{X}_{m}^{\prime}\right] / I$ by dividing out by a regular 
sequence of variable differences $x_{i a}-x_{i b}$ where for each $i, x_{i a}$ and $x_{i b}$ are in the same fiber $p_{i}^{-1}\left(x^{\prime}\right)$ of $p_{i}$, and we have $\left|p_{i}^{-1}\left(x^{\prime}\right)\right|-1$ linearly independent such variable differences for each fiber.

The following lemma has some key consequences.

Lemma 2.5. Let $x^{\mathbf{a}}$ and $x^{\mathbf{b}}$ be minimal generators of a monomial ideal $I$ and $m(\mathbf{a})$ and $m(\mathbf{b})$ the corresponding generators in a polarization of $I$.

Fix an index $i$. If $a_{i} \leq b_{i}$ and $a_{j} \geq b_{j}$ for every $j \neq i$, then the $i$ 'th part $m_{i}(\mathbf{a})$ divides $m_{i}(\mathbf{b})$.

Proof. We shall use induction on $d=b_{i}-a_{i}$. If $d=0$ then clearly $\mathbf{b}=\mathbf{a}$ and there is nothing to prove. We may also assume that $a_{i} \geq 1$, since otherwise there is nothing to prove. We suppose $m_{i}(\mathbf{a})$ does not divide $m_{i}(\mathbf{b})$ and we shall derive a contradiction. If it does not divide we may factor $m_{i}(\mathbf{b})$ as $t_{i}(\mathbf{b}) \cdot n_{i}(\mathbf{b})$ where $t_{i}(\mathbf{b})$ has degree $d+1$ and has no common variable with $m_{i}(\mathbf{a})$. (We are of course using here that $m_{i}(\mathbf{b})$ is squarefree.) For simplicity we may re-index variables so that $t_{i}(\mathbf{b})=x_{i 1} x_{i 2} \cdots x_{i, d+1}$. We now in $k\left[\check{X}_{1}, \ldots, \check{X}_{m}\right] / J$ divide out by all the variable differences involving $\check{X}_{j^{-}}$ variables where $j \neq i$, and by all variable differences $x_{i r}-x_{i, r+1}$ for $r=d+2, \ldots, n_{i}-1$. Thus we are collapsing all the $\check{X}_{j}$-variables into the single variable $x_{j}$ and the variables $x_{i, d+2}, \ldots, x_{i, n_{i}}$ into a single variable $x_{i}$. We get a quotient ring

$$
k\left[x_{i 1}, \ldots, x_{i, d+1}, x_{1}, \ldots, x_{m}\right] / I^{\prime},
$$

where $I^{\prime}$ is a separation of $I$. Note that $m(\mathbf{a})$ collapses to $x^{\mathbf{a}}$ in $I^{\prime}$.

Consider now the variable difference $x_{i, d+1}-x_{i}$ in the polynomial ring above. We see that

$$
\begin{aligned}
& \left(x_{i, d+1}-x_{i}\right) x_{i 1} \cdots x_{i d} \cdot x_{i}^{a_{i}-1} \prod_{j \neq i} x_{j}^{a_{j}} \\
= & x_{i 1} \cdots x_{i, d+1} \cdot x_{i}^{a_{i}-1} \prod_{j \neq i} x_{j}^{a_{j}}-x_{i 1} \cdots x_{i, d} \cdot x_{i}^{a_{i}} \prod_{j \neq i} x_{j}^{a_{j}}
\end{aligned}
$$

vanishes in the quotient ring (5): Tthe first term is divisible by the image of $m(\mathbf{b})$ in $I^{\prime}$ (note that $b_{i}=(d+1)+\left(a_{i}-1\right)$ ), and the second term is divisible by the image of $m(\mathbf{a})$. Since $x_{i, d+1}-x_{i}$ is not a zero divisor (it belongs to a regular sequence), we get from (6) that

$$
\mathbf{n}=x_{i 1} \cdots x_{i d} \cdot x_{i}^{a_{i}-1} \prod_{j \neq i} x_{j}^{a_{j}}
$$

is in $I^{\prime}$. Now if $d=1$, this monomial has $\mathbb{Z}^{m}$-degree a. But the monomial $x^{\mathbf{a}}$ is in $I^{\prime}$, with the same degree. Since these are the $\mathbb{Z}^{m}$-degree of a generator of $I$, there can only be a single monomial in $I^{\prime}$ with this $\mathbb{Z}^{m}$-degree. We get a contradiction. Now suppose $d \geq 2$. Then $\mathbf{n}$ is divisible by a generator $m^{\prime}(\mathbf{c})$ in $I^{\prime}$ which can not be $x^{\mathbf{a}}$. We will have each $c_{j} \leq a_{j}$ for $j \neq i$, and so $c_{i}>a_{i}$. Furthermore, we have $b_{i}>c_{i}$ since $\mathbf{n}$ in (77) has $i$-degree $d+a_{i}-1=b_{i}-1$. By induction on $d$, considering the polarized ideal $J$, the $i$ 'th part $m_{i}(\mathbf{a})$ here divides the $i^{\prime}$ th part $m_{i}(\mathbf{c})$. But then going to $I^{\prime}$ then $x_{i}^{a_{i}}$ divides the image of $m_{i}^{\prime}(\mathbf{c})$, and so $x_{i}^{a_{i}}$ would divide $\mathbf{n}$ of (7), a contradiction. 
Remark 2.6. If $m(\mathbf{a})$ is a minimal generator of $J$, by the lemma $m_{i}(\mathbf{a})$ will divide $m_{i}\left(0, \ldots, n_{i}, \ldots, 0\right)$ which of course is just $m\left(0, \ldots, n_{i}, \ldots, 0\right)$. Thus, if the polarization of $x_{i}^{n_{i}}$ is $x_{i 1} x_{i 2} \cdots x_{i n_{i}}$, then every $x_{i}$-variable occurring in the minimal generators of $J$ are among these variables, and so we may take $\check{X}_{i}=\left\{x_{i 1}, \ldots, x_{i n_{i}}\right\}$.

The following is quite particular for Artinian monomial ideals, note Example 2.2.

Corollary 2.7. Every polarization of an Artinian monomial ideal $I$ is a separated model for $I$.

Proof. If the polarization $J$ was not a separated model, then let $J^{\prime}$ be a further simple separation. Since $I$ in $k\left[x_{1}, \ldots, x_{m}\right]$ is an Artinian monomial ideal, every variable $x_{i}$ of course occurs in a minimal generator of $I$, in fact $x_{i}^{n_{i}}$ is a minimal generator. Then if $J^{\prime}$ is in $k\left[\check{X}_{1}^{\prime}, \ldots, \check{X}_{m}^{\prime}\right]$ then every variable in this polynomial ring must also occur in a generator of $J^{\prime}$, by the definition of a separation. By the above Lemma 2.5 and Remark 2.6, if $x_{i}^{n_{i}}$ polarizes to $x_{i 1} \cdots x_{i n_{i}}$ then $\check{X}_{i}^{\prime}=\left\{x_{i 1}, \ldots, x_{i n_{i}}\right\}$. But $J$ is obtained from $J^{\prime}$ by dividing out by a variable difference $x_{i a}-x_{i b}$. Then the image of $x_{i 1} \cdots x_{i n_{i}}$ in $J$ would not be squarefree, a contradiction.

2.3. Polarizations of powers of the graded maximal ideal. We now consider powers of the maximal ideals

$$
M=\left(x_{1}, x_{2}, \ldots, x_{m}\right)^{n} \subseteq k\left[x_{1}, \ldots, x_{m}\right] .
$$

A polarization of this ideal may by Remark 2.6 be taken to live in polynomial ring $k\left[\check{X}_{1}, \ldots, \check{X}_{m}\right]$ where $\check{X}_{i}=\left\{x_{i 1}, \ldots, x_{i n}\right\}$. Our goal is to combinatorially classify all possible polarizations of $M$ in this polynomial ring.

The generators of the monomial ideal $M$ are all monomials $x_{1}^{b_{1}} \ldots x_{m}^{b_{m}}$ with $b_{1}+\cdots+$ $b_{m}=n$.

Definition 2.8. $\Delta_{m}(n)$ is the subset of $\mathbb{N}_{0}^{m}$ of all tuples $\mathbf{b}=\left(b_{1}, \ldots, b_{m}\right)$ of non-negative integers with $b_{1}+\cdots+b_{m}=n$. For a given $\mathbf{b}$, its support supp (b) is the set of all $i$ such that $b_{i} \geq 1$.

In a polarization $J$ of $M$ we have one minimal generator of $J, m(\mathbf{b})$ for every $\mathbf{b}$ in $\Delta_{m}(n)$. Now fix an index $1 \leq i \leq m$. Then $\Delta_{m}(n)$ may be given a partial order $\geq_{i}$ by letting $\mathbf{b} \geq_{i}$ a if $b_{i} \geq a_{i}$ and $b_{j} \leq a_{j}$ for $j \neq i$. Thus there is one maximal element $(0, \ldots, 0, n, 0, \ldots, 0)$ where $n$ is in position $i$, and it has minimal elements all $\mathbf{b}$ with $b_{i}=0$. This is a graded partial order with $\mathbf{b}$ of rank $b_{i}$.

Now, given any $\mathbf{b} \in \Delta_{m}(n)$, we get from the polarization $J$ a squarefree monomial $m_{i}(\mathbf{b})$, see (4). The set of variables dividing this monomial is a subset of $\check{X}_{i}$ which we denote as $X_{i}(\mathbf{b})$. Let $B\left(\check{X}_{i}\right)$ be the Boolean poset on $\check{X}_{i}$, a Boolean poset on a set of $n$ elements. We get a function

$$
\begin{aligned}
X_{i}: \Delta_{m}(n) & \rightarrow B\left(\check{X}_{i}\right) \\
\mathbf{b} & \mapsto X_{i}(\mathbf{b}) .
\end{aligned}
$$

The following is an immediate consequence of Lemma 2.5.

Corollary 2.9. Let $J$ be a polarization of $M$. Then $X_{i}$ is an isotone rank-preserving map when $\Delta_{m}(n)$ has the ordering $\geq_{i}$. 


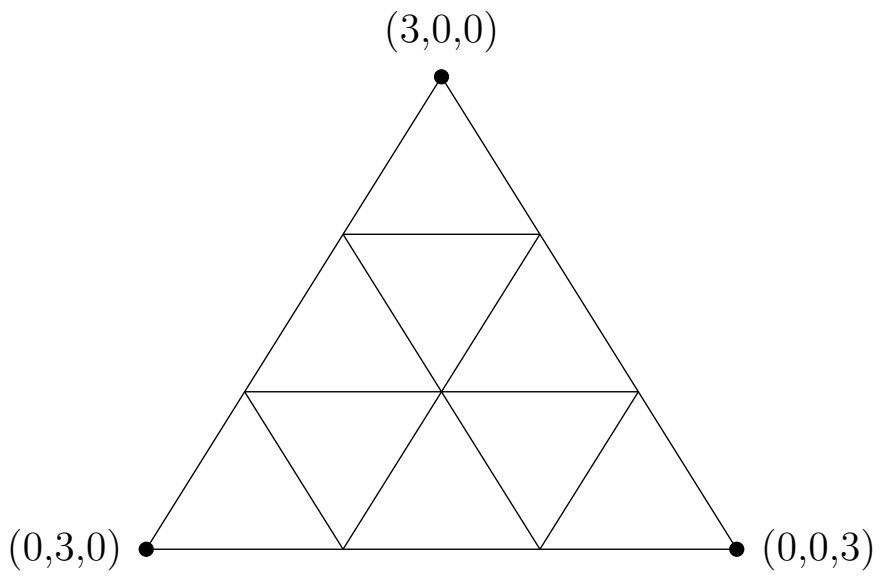

FiguRE 2 .

Remark 2.10. Since $\check{X}_{i}=\left\{x_{i 1}, \ldots, x_{i n}\right\}$, the group $S_{n}$ acts on $\check{X}_{i}$. Also the group $S_{m}$ acts on $k\left[x_{1}, \ldots, x_{m}\right]$ by permutation of variables and hence on the set of maps $\left\{X_{i}\right\}$. In all there is an action of a semi-direct product $S_{m} \ltimes\left(S_{n}\right)^{m}$ on $k\left[\check{X}_{1}, \ldots, \check{X}_{m}\right]$ compatible with the action of $S_{m}$ on $k\left[x_{1}, \ldots, x_{m}\right]$. Since $\left(x_{1}, \ldots, x_{m}\right)^{n}$ is equivariant for the group action, the isomorphism classes of polarizations of this maximal ideal power are precisely the orbits of $S_{m} \ltimes\left(S_{n}\right)^{m}$ on the set of polarizations.

The convex hull of $\Delta_{m}(n)$ in $\mathbb{R}^{m}$ is a simplex of dimension $(m-1)$. We shall however only need the graph structure it induces. Given a point $\mathbf{c}$ in $\Delta_{m}(n+1)$ and $i, j$ in the support of $\mathbf{c}$, let $e_{i}$ and $e_{j}$ be the unit coordinate vectors. Then we get an edge between the points $\mathbf{c}-e_{i}$ and $\mathbf{c}-e_{j}$ in $\Delta_{m}(n)$, denoted $(\mathbf{c} ; i, j)$. Every edge in $\Delta_{m}(n)$ is of this form for unique $\mathbf{c}, i$ and $j$. A point $\mathbf{c}$ of $\Delta_{m}(n+1)$ induces a subgraph of $\Delta_{m}(n)$, the complete down-graph $D(\mathbf{c})$ on the points $\mathbf{c}-e_{i}$ for $i \in \operatorname{supp}(\mathbf{c})$. The dimension of $D(\mathbf{c})$ is the dimension of its convex hull, which is one less than the cardinality of supp (c). When supp (c) has dimension two we call this a down-triangle. In Figure 2 we have three down-triangles.

Let $\Delta_{m}^{+}(n+1)$ be the subset of $\Delta_{m}(n+1)$ consisting of $\mathbf{c}$ with $c_{i} \geq 1$ for every $i$. The complete down-graph $D(\mathbf{c})$ has full (maximal) dimension $(m-1)$ iff $\mathbf{c} \in \Delta_{m}^{+}(n+1)$. Note that $\Delta_{m}^{+}(n+1)$ is in one-one correspondence with $\Delta_{m}(n+1-m)$ by sending $\mathbf{c}$ to $\mathbf{c}-\mathbf{1}$ where $\mathbf{1}=(1,1, \ldots, 1)$. In Figure 2 there are three full-dimensional complete down-graphs, or in this case down-triangles, corresponding to the three elements of $\Delta_{3}(1)$, or equivalently of $\Delta_{3}^{+}(4)$.

Each a in $\Delta_{m}(n-1)$ also determines a subgraph of $\Delta_{m}(n)$, the complete up-graph $U(\mathbf{a})$ consisting of the points $\mathbf{a}+e_{i}$ for $i=1, \ldots, m$ and with edges $\left(\mathbf{a}+e_{i}+e_{j} ; i, j\right)$ for $i \neq j$. For each a in $\Delta_{m}(n-1)$ the convex hull of the up-graph $U(\mathbf{a})$ has full dimension $(m-1)$. When $m=3$ we call this an up-triangle. In Figure 2 there are six up-triangles. 


\section{Conjectures And PRoblems}

Before embarking on the main results of the paper we here discuss conjectures and problems on polarizations of Artinian monomial ideals in general that have come up during our investigations. They concern i) the topology of their associated simplicial complexes, ii) their Alexander duals, a class of rainbow monomial ideals (terminology introduced here), iii) their deformations.

\subsection{Balls and spheres.}

Lemma 3.1. Let $\Delta(J)$ be the simplicial complex associated to the polarization $J$ of an Artinian monomial ideal $I$. Then every codimension one face of $\Delta(J)$ is contained in one or two facets. If I is not a complete intersection, then at least once there is a codimension one face contained in exactly one facet.

Proof. Let $\Delta_{i}$ be the simplex on $\left\{(i, j) \mid j=1, \ldots, n_{i}\right\}$. The squarefree monomial $x_{i 1} x_{i 2} \cdots x_{i n_{i}}$ in $k\left[\check{X}_{i}\right]$ defines the sphere which is the boundary $\partial \Delta_{i}$ of this simplex. The natural polarization in $k\left[\check{X}_{1}, \ldots, \check{X}_{m}\right]$ of the complete intersection $\left(x_{1}^{n_{1}}, x_{2}^{n_{2}}, \ldots, x_{m}^{n_{m}}\right)$ then defines the sphere of codimension $m$ which is the join $S=\underset{i=1}{m} \partial \Delta_{i}$. Every codimension one face is here on precisely two facets.

The simplicial complex $\Delta(J)$ is a Cohen-Macaulay subcomplex of $S$ with the same dimension as $S$. If $\Delta(J)$ is not all of $S$, let $F$ be a facet of $\Delta(J)$ and $G$ a facet of $S$ not in $\Delta(J)$. Since $S$ is strongly connected, there is a path of facets

$$
F=F_{0}, F_{1}, \ldots, F_{r}=G
$$

such that $F_{i} \cap F_{i+1}$ has codimension one for each $i$, [17, Prop.9.1.12]. Let $p$ be maximal such that $F_{p}$ is in $\Delta(J)$. Then $F_{p} \cap F_{p+1}$ is only on the facet $F_{p}$ in $\Delta(J)$.

By a result of Björner [4, Thm.11.4] a constructible simplicial complex with the property of Lemma 3.1 above is a simplicial ball or a simplicial sphere (the latter when every codimension one face is on exactly two facets).

Conjecture 3.2. The simplicial complex $\Delta(J)$ associated to a polarization $J$ of an Artinian monomial ideal $I$, is a simplicial ball, save for the case when $I$ is a complete intersection, when it is a simplicial sphere.

By the result of Björner loc.cit. a positive answer to the following question would settle the above conjecture.

Question 3.3. Do polarizations of Artinian monomial ideals have constructible (for instance shellable) simplicial complexes?

That the standard polarization of an Artinian monomial ideal is shellable seems first to have been shown by A.Soleyman Jahan in [25]. In [22] S.Murai uses this to conclude that the standard polarizations give simplicial balls. More generally it is shown that letterplace ideals define simplicial balls, [7, by showing that these simplicial complexes are shellable. Letterplace ideals are introduced in [13] and are polarizations of Artinian monomial ideals. The article [12] discusses such Artinian monomial ideals more in depth. 
In our last Section 8 we show in the case of three variables that the Alexander dual of any polarization $J$ has linear quotients, see [17, Sec.8.2, Cor.8.2.4] for this notion. In Section 6 we show when the power of the maximal ideal (in any number of variables) is two, then the Alexander dual has linear quotients. Thus in these cases the simplicial complex $\Delta(J)$ is shellable and hence a simplicial ball.

For a letterplace ideal the second author et.al. in [7] get an explicit simple description of the Stanley-Reisner ideal of the boundary of the simplicial ball defined by the letterplace ideal. In fact a general result, see [6, Section 5], says that the canonical module of a Stanley-Reisner ring $k[\Delta]$ identifies as a multigraded proper ideal of this ring $k[\Delta]$ if and only if $\Delta$ is a homology ball. Then the ideal defines the boundary of this homology ball, which is a homology sphere. In [7] an explicit description of this canonical module is given.

Conjecture 3.4. For polarizations of Artinian monomial ideals, the canonical module identifies (in a simply described and natural way) as a multigraded ideal of the StanleyReisner ring of the polarization.

Consequence 3.5. With Conjecture 3.2, this would give an explicit description of the Stanley-Reisner ring of the boundary of the simplicial ball, defining a simplicial sphere.

3.2. Rainbow monomial ideals with linear resolution. Recall that for a squarefree monomial ideal $J$ in a polynomial ring $S$, the squarefree monomial ideal $I$ is the Alexander dual of $J$ if the monomials in $I$ are precisely the monomials in $S$ which have nontrivial common divisor with every monomial in $J$, or equivalently, every generator of $J$.

We may consider each set of variables $\check{X}_{i}, i=1, \ldots, m$ as a color class of monomials. A monomial $x_{1 i_{1}} x_{2 i_{2}} \cdots x_{m i_{m}}$ with one variable of each color is a rainbow monomial.

Proposition 3.6. The class of ideals generated by rainbow monomials and with $m$ linear resolution is precisely the class which is Alexander dual to the class of polarizations of Artinian monomial ideals in $m$ variables:

a. Let $J$ be a polarization of an Artinian monomial ideal $I$ in $k\left[x_{1}, \ldots, x_{m}\right]$. The Alexander dual ideal of $J$ is generated by rainbow monomials and has $m$-linear resolution.

b. If an ideal $J^{\prime}$ is generated by rainbow monomials and has m-linear resolution (and every variable in the ambient ring occurs in some generator of the ideal), then its Alexander dual $J$ is a polarization of an Artinian monomial ideal in $m$ variables.

Proof. a. Since $I$ is Cohen-Macaulay of codimension $m$, the same is true for $J$. Then the Alexander dual of $J$ is generated in degree $m$ and has $m$-linear resolution [8]. But if $\mathbf{m}$ is a generator for this Alexander dual, it has a common variable with $x_{i 1} x_{i 2} \cdots x_{i n_{i}}$ (the polarization of $x_{i}^{n_{i}}$ ) for every $i=1, \ldots, m$. Hence $\mathbf{m}$ must have a variable of each of the $m$ colors.

b. By [8], the Alexander dual $J$ of $J^{\prime}$ is Cohen-Macaulay of codimension $m$. For each color class $\check{X}_{i}=\left\{x_{i 1}, \ldots, x_{i n_{i}}\right\}$, the ideal $J$ will contain the monomial which is the product of all these variables. 
If we for every color class $i$ divide the quotient $\operatorname{ring} k\left[\check{X}_{1}, \ldots, \check{X}_{m}\right] / J$ by all the variable differences $x_{i, j}-x_{i, j-1}$ for $j=2, \ldots, n_{i}$, we get a quotient ring $k\left[x_{1}, \ldots, x_{m}\right] / I$ where $I$ is Artinian since it contains $x_{i}^{n_{i}}$ for each $i$. Hence like $J$ the ideal $I$ is Cohen-Macaulay of codimension $m$. Then the sequence we divided out by must have been a regular sequence, and so $J$ is a polarization of $I$.

Remark 3.7. Considering the $\check{X}_{i}$ as color classes, both the Artinian ideal $I$, the polarization $J$ and its Alexander dual are generated by colored monomials. Such ideals and the associated simplicial complexes have been considered in various settings, like balanced simplicial complexes by Stanley [26], relating to the colorful topological Helly theorem by Kalai and Meshulam, [19], and resolutions of such ideals by the second author [11].

In Section 7 we describe the Alexander dual of any polarization $J$ of a maximal ideal power. But while the description of the generators of $J$ is rather direct, Sections 4 and 6. the description the Alexander dual is more subtle. For instance, it is not obvious from the description that there are actually always $\left(\begin{array}{c}n+m-1 \\ m\end{array}\right)$ generators of the Alexander dual.

In [24] A. Nematbakhsh gives a precise description of when an ideal generated by rainbow monomials has linear resolution. His terminology for rainbow monomials of $d$ colors is "edge monomials of $d$-partite $d$-uniform clutters". He is able to give a characterization through a remarkable connection to the article [9] where they give a characterization of when a point set in the multiprojective space $\left(\mathbb{P}^{1}\right)^{n}$ is arithmetically Cohen-Macaulay (meaning that the associated multihomogeneous coordinate ring is a Cohen-Macaulay ring). The characterization given in [24] by translating the one in [9] is the following.

Theorem 3.8. Let I be generated by rainbow monomials in d colors. Then I has a d-linear resolution iff:

a. Whenever $m_{1}$ and $m_{2}$ are two rainbow monomials in $I$ (i.e. generators of degree d) with $\operatorname{lcm}\left(m_{1}, m_{2}\right)$ of degree $\geq d+2$, there is a third distinct rainbow monomial $m_{3}$ in $I$ dividing this least common multiple.

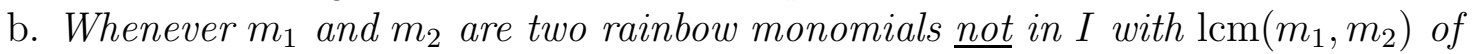
degree $\geq d+2$, there is a third distinct rainbow monomial $m_{3}$ not in I dividing this least common multiple.

So this says that a subset $A$ of the product $\check{X}_{1} \cdot \check{X}_{2} \cdots \check{X}_{d}$ gives an ideal with $d$-linear resolution iff both $A$ and its complement are in some sense convex.

Fröberg's theorem [15] characterizes when a monomial ideal generated in degree two has linear resolution. It is easily seen that both this theorem and the theorem above give the following criterion when we have rainbow monomials with two colors: If $x_{a} y_{b}$ and $x_{a^{\prime}} y_{b^{\prime}}$ are in $I$, then either $x_{a} y_{b^{\prime}}$ or $x_{a^{\prime}} y_{b}$ is in $I$. Many attempts have been done to generalize Fröberg's theorem to higher degrees, but none fully successful. For rainbow monomials, however, the above gives such a generalization.

Example 3.9. Let $X=\left\{x_{1}, x_{2}\right\}, Y=\left\{y_{1}, y_{2}\right\}$ and $Z=\left\{z_{1}, z_{2}\right\}$ be three color classes and let $I$ be the ideal generated by the six monomials

$$
x_{1} y_{1} z_{2}, x_{1} y_{2} z_{1}, x_{2} y_{1} z_{1}, x_{2} y_{2} z_{1}, x_{2} y_{1} z_{2}, x_{1} y_{2} z_{2} .
$$


Then $I$ does not have linear resolution since $x_{1} y_{1} z_{1}$ and $x_{2} y_{2} z_{2}$ are not in $I$ and their least common multiple is not divided by any distinct rainbow monomial not in $I$.

If we remove $x_{1} y_{1} z_{2}$ we also do not have linear resolution, since now the least common multiple of $x_{2} y_{1} z_{2}$ and $x_{1} y_{2} z_{2}$ is not divided by any distinct rainbow monomial in $I$. If we remove both $x_{1} y_{1} z_{2}$ and $x_{1} y_{2} z_{2}$ then we do get a monomial ideal with linear resolution.

Problem 3.10. Consider an ideal $J^{\prime}$ generated by rainbow monomials. Is there a direct criterion on this ideal to tell if its Alexander dual is a polarization of a power of a graded maximal ideal?

Since an Artinian monomial ideal has linear resolution iff it is a power of the graded maximal ideal, this is the same as asking for a criterion for the ideal $J^{\prime}$ to be bi-CohenMacaulay: The ideal has both linear resolution and is Cohen-Macaulay.

Such a description would maybe involve reconstructing the isotone maps $X_{i}$ given in (8), from $J^{\prime}$. In [3] a cellular resolution is computed when $J^{\prime}$ is the Alexander dual of the standard polarization $J$ of any Artinian monomial ideal.

Remark 3.11. If we for each color class $i$ have only two variables $\left\{x_{i 0}, x_{i 1}\right\}$, then a rainbow monomial of degree $m$ may be identified with a binary string, say if $m=6$ then 101011 corresponds to $x_{11} x_{20} x_{31} x_{40} x_{51} x_{61}$. Thus investigating homological properties of ideals generated by rainbow monomials with two variables of each color, corresponds to investigating algebraic and topological properties of sets of binary words.

3.3. Deformations of polarizations. In [14 the second author and A.Nematbakhsh showed that the letterplace ideals $L(2, P)$ (which are polarizations of quadratic Artinian monomial ideals) have unobstructed deformations when the Hasse diagram is a tree. Moreover we computed the full deformation family of these ideals. Together with G.Scattareggia we have also verified that all deformations of various polarizations of quadratic powers $\left(x_{1}, x_{2}, \ldots, x_{m}\right)^{2}$ lift to global deformations for $m=3,4$ by computing a full global family. We have also verified this for the letterplace ideal $L(3,5)$ (introduced in [13]), a cubic ideal.

Conjecture 3.12. Every first order deformation of a polarization of an Artinian monomial ideal (regardless of whether the deformation is homogeneous for the standard grading) lifts to a global deformation, with any grading on the first order deformation respected by the global deformation. In particular, whenever such a polarization is on a (multigraded) Hilbert scheme, it is a smooth point.

If the homogeneous ideal $I$ in the polynomial ring $S$ corresponds to a point on a Hilbert scheme of subschemes of a projective space, then the tangent space of the Hilbert scheme at this point is the degree zero part $\operatorname{Hom}_{S}(I, S / I)_{0}$. By explicit computation with Macaulay 2, one can verify that the dimension of this space varies quite much for different polarizations. For instance, for the polarizations of $\left(x_{1}, x_{2}, x_{3}\right)^{3}$, the lowest dimension of the tangent space occurs for the standard polarization, with dimension 69. The largest dimension occurs for the b-polarization, giving a dimension of 102 (this ideal is a smooth point on the Hilbert scheme component of ideals of maximal minors of $3 \times 5$ matrices of linear forms). Furthermore there are also many values of the dimension of this tangent space between 69 and 102. Thus if they are all smooth points, they would be on many different components of the Hilbert scheme. 

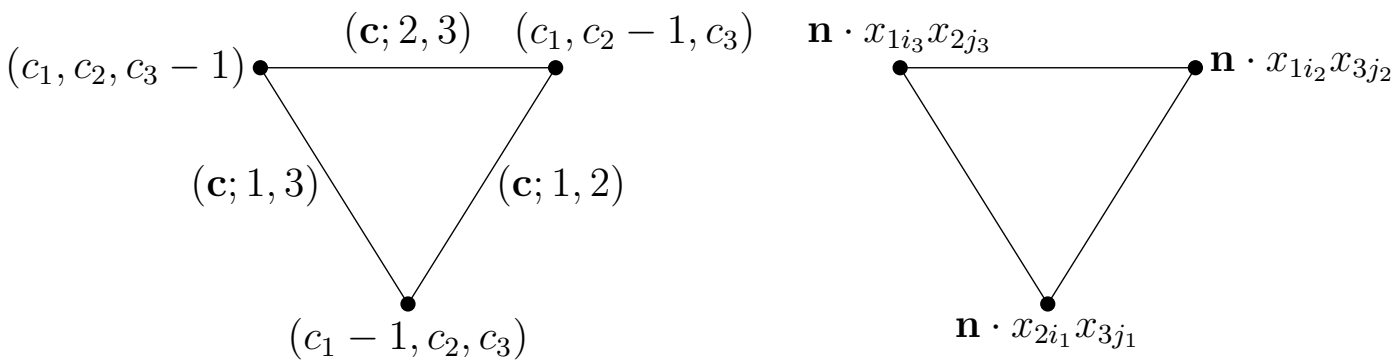

FIGURE 3.

\section{Polarizations and linear Syzygy edges}

We here give our main result, the complete combinatorial description, Theorem 4.5, of all polarizations of powers of maximal ideals $\left(x_{1}, \ldots, x_{m}\right)^{n}$. Write $[m]=\{1,2, \ldots, m\}$.

4.1. Statement and examples. The essential objects are the rank-preserving isotone maps $X_{i}: \Delta_{m}(n) \rightarrow B(n)$ for $i=1, \ldots, m$ and conditions on them. For each $\mathbf{b} \in \Delta_{m}(n)$ we get a monomial $m_{i}(\mathbf{b})=\prod_{j \in X_{i}(\mathbf{b})} x_{i j}$ in the variables $\check{X}_{i}=\left\{x_{i 1}, \ldots, x_{i n}\right\}$. To the vertex $\mathbf{b}$ we associate the monomial

$$
m(\mathbf{b})=\prod_{i=1}^{m} m_{i}(\mathbf{b})
$$

and let $J$ be the ideal in $k\left[\check{X}_{1}, \ldots, \check{X}_{m}\right]$ generated by the $m(\mathbf{b})$.

If $B$ is a subset of $[m]$, denote by $\mathbf{1}_{B}$ the $m$-tuple $\sum_{i \in B} e_{i}$. For instance, if $B=[m]$, then $\mathbf{1}_{B}=(1,1, \ldots, 1)$.

Lemma 4.1. Let $\mathbf{c} \in \Delta_{m}(n+1)$ have support $C \subseteq\{1,2, \ldots, m\}$. The monomials associated to the vertices in the down-graph $D(\mathbf{c})$ have a common factor of degree $\mathbf{c}-\mathbf{1}_{C}$. This common factor is $\prod_{i \in C} m_{i}\left(\mathbf{c}-e_{i}\right)$.

Proof. Fix an element $j \in C$. For the order $\geq_{k}$ we have $\mathbf{c}-e_{j} \geq_{k} \mathbf{c}-e_{k}$ for every $k \in C$. Hence $X_{k}\left(\mathbf{c}-e_{k}\right)$ is contained in $X_{k}\left(\mathbf{c}-e_{j}\right)$ for every $k \in C$. Thus $m\left(\mathbf{c}-e_{j}\right)$ has $m_{k}\left(\mathbf{c}-e_{k}\right)$ as a factor for each $k \in C$.

Example 4.2. Let $m=3$ and $\mathbf{c}=\left(c_{1}, c_{2}, c_{3}\right)$ be in $\Delta_{3}^{+}(n+1)$. At the left in Figure 3 we illustrate the down triangle $D(\mathbf{c})$. Let

$$
\mathbf{n}=m_{1}\left(\mathbf{c}-e_{1}\right) \cdot m_{2}\left(\mathbf{c}-e_{2}\right) \cdot m_{3}\left(\mathbf{c}-e_{3}\right) .
$$

Then the monomials associated to the vertices of this down-triangle are shown to the right in Figure 3 ,

Definition 4.3. An edge $(\mathbf{c} ; i, j)$ in $\Delta_{m}(n)$ (where $\mathbf{c} \in \Delta_{m}(n+1)$ ) is a linear syzygy edge (LS-edge) if there is a monomial $\mathbf{m}$ of degree $(n-1)$ such that

$$
m\left(\mathbf{c}-e_{i}\right)=x_{j r} \cdot \mathbf{m}, \quad m\left(\mathbf{c}-e_{j}\right)=x_{i s} \cdot \mathbf{m}
$$

for suitable variables $x_{j r} \in \check{X}_{j}$ and $x_{i s} \in \check{X}_{i}$. So this edge gives a linear syzygy between the monomials $m\left(\mathbf{c}-e_{i}\right)$ and $m\left(\mathbf{c}-e_{j}\right)$. By Lemma 4.1 both $m_{i}\left(\mathbf{c}-e_{i}\right)$ and $m_{j}\left(\mathbf{c}-e_{j}\right)$ 
are common factors of $m\left(\mathbf{c}-e_{i}\right)$ and $m\left(\mathbf{c}-e_{j}\right)$. So (9) is equivalent to

$$
m_{p}\left(\mathbf{c}-e_{i}\right)=m_{p}\left(\mathbf{c}-e_{j}\right) \text { for every } p \neq i, j,
$$

or formulated in terms of the isotone maps

$$
X_{p}\left(\mathbf{c}-e_{i}\right)=X_{p}\left(\mathbf{c}-e_{j}\right) \text { for every } p \neq i, j .
$$

We denote by LS(c) the set of linear syzygy edges in the complete down-graph $D(\mathbf{c})$.

Example 4.4. In Figure 3 the edge $(\mathbf{c} ; 2,3)$ is a linear syzygy edge when $x_{1 i_{3}}=x_{1 i_{2}}$. Similarly the edge $(\mathbf{c} ; 1,3)$ is a linear syzygy edge when $x_{2 j_{3}}=x_{2 i_{1}}$. In general, when the support of $\mathbf{c}$ has cardinality three and when $(\mathbf{c} ; i, j)$ is not a linear syzygy edge we call it a quadratic syzygy $(Q S)$ edge, since we then have a quadatic syzygy between the monomials $m\left(\mathbf{c}-e_{i}\right)$ and $m\left(\mathbf{c}-e_{j}\right)$

Here is the main theorem of this article.

Theorem 4.5. The isotone maps $X_{1}, \ldots, X_{m}$ determine a polarization of the ideal $\left(x_{1}, \ldots, x_{m}\right)^{n}$ if and only if for every $\mathbf{c} \in \Delta_{m}(n+1)$, the linear syzygy edges LS(c) contain a spanning tree for the down-graph $D(\mathbf{c})$.

We prove this towards the end of this section.

Remark 4.6. For every $\mathbf{c} \in \Delta_{m}(n+1)$ which has support $\{i, j\}$ of cardinality 2 , it is automatic by the condition that the $X_{p}$ are rank-preserving and isotone that the edge $(\mathbf{c} ; i, j)$ is a linear syzygy edge in LS(c). (Condition (10) is empty.) So the conditions in Theorem 4.5 is automatically fulfilled for the $\mathbf{c}$ with support of cardinality 2.

Corollary 4.7. When $m=3$ the isotone maps $X_{1}, X_{2}, X_{3}$ give a polarization of $\left(x_{1}, x_{2}, x_{3}\right)^{n}$ iff each down-triangle contains at most one QS-edge.

Example 4.8. Write $x, y, z$ for $x_{1}, x_{2}, x_{3}$. Consider the ideal

$$
J=\left(x_{1} x_{2} x_{3}, x_{1} x_{2} y_{2}, x_{1} x_{2} z_{2}, x_{2} y_{1} y_{2}, x_{1} y_{1} z_{2}, x_{1} z_{1} z_{2}, y_{1} y_{2} y_{3}, y_{1} y_{2} z_{2}, y_{1} z_{2} z_{3}, z_{1} z_{2} z_{3}\right)
$$

which is a polarization of $(x, y, z)^{3} \subset k[x, y, z]$. In Figure 4 we denote quadratic syzygy edges by dashed lines. We see that each of the three down-triangles has exactly one quadratic syzygy edge.

Proof of Corollary 4.7. A $\mathbf{c} \in \Delta_{3}(n+1)$ has support of cardinality 1,2 or 3 . If it is 3 , we have a down-triangle, and so at least two of the three edges must be linear syzygy edges. Equivalently at most one of the edges is a QS-edge. If the cardinality is 2, the edge is on the boundary of the simplex induced by $\Delta_{3}(n)$ and it is a linear syzygy edge by the above Remark 4.6.

4.2. Linear syzygy paths. Let $R \subseteq[m]$ and $\mathbf{c} \in \Delta_{m}(n+1)$ with $R$ contained in the support of $\mathbf{c}$. Let $r, s \in R$. We say $(\mathbf{c} ; r, s)$ is an $R$-linear syzygy edge if

$$
X_{p}\left(\mathbf{c}-e_{r}\right)=X_{p}\left(\mathbf{c}-e_{s}\right) \text { for } p \in R \backslash\{r, s\} .
$$

Take note that by isotonicity of the $X_{p}$, for $p=r, s$ :

$$
X_{r}\left(\mathbf{c}-e_{r}\right) \subseteq X_{r}\left(\mathbf{c}-e_{s}\right), \quad X_{s}\left(\mathbf{c}-e_{s}\right) \subseteq X_{s}\left(\mathbf{c}-e_{r}\right)
$$

Let $D_{R}(\mathbf{c})$ be the complete graph with edges $(\mathbf{c} ; r, s)$ for $r, s \in R$. 


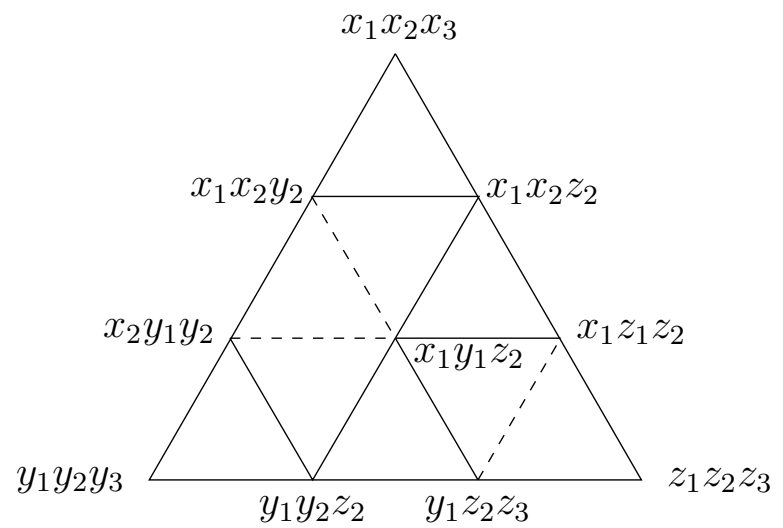

FiguRE 4.
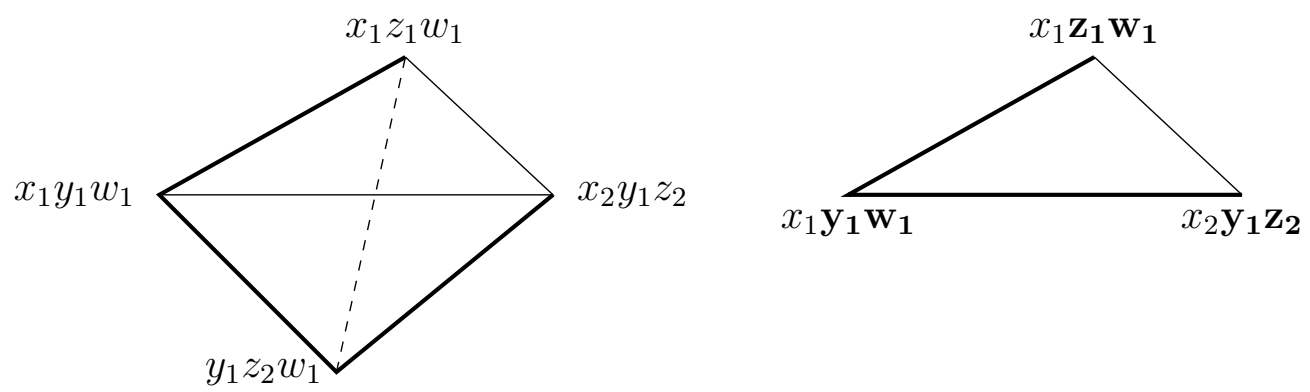

FiguRE 5 .

Lemma 4.9. Let $\mathbf{c} \in \Delta_{m}(n+1)$. If the set of linear syzygy edges in $\mathrm{LS}(\mathbf{c})$ contains a spanning tree for $D(\mathbf{c})$, then for each $R \subseteq \operatorname{supp}(\mathbf{c})$, the set of $R$-linear syzygy edges contains a spanning tree for $D_{R}(\mathbf{c})$.

Example 4.10. Consider the case of four variables and $\mathbf{c}=(1,1,1,1)$. Write $x, y, z, w$ for $x_{1}, x_{2}, x_{3}, x_{4}$. On the left of Figure 5 is the down-graph $D(\mathbf{c})$ with the three thick edges the linear syzygy edges.

Let $R=\{2,3,4\}$. On the right is the down-graph $D_{R}(\mathbf{c})$ where the two thick edges are the $R$-linear syzygy edges and the relevant variables marked in bold.

Proof. Let $Q$ be the complement of $R$ in $\operatorname{supp}(\mathbf{c})$. Let $r$ and $s$ be two elements in $R$. There is a path from $\mathbf{c}-e_{r}$ to $\mathbf{c}-e_{s}$ in $D(\mathbf{c})$ consisting of linear syzygy edges. It may be broken up into smaller paths: From $\mathbf{c}-e_{r}=\mathbf{c}-e_{r_{0}}$ to $\mathbf{c}-e_{r_{1}}$, from $\mathbf{c}-e_{r_{1}}$ to $\mathbf{c}-e_{r_{2}}$, $\ldots$, from $\mathbf{c}-e_{r_{p-1}}$ to $\mathbf{c}-e_{r_{p}}=\mathbf{c}-e_{s}$ where on the path from $\mathbf{c}-e_{r_{i-1}}$ to $\mathbf{c}-e_{r_{i}}$ the only vertices $\mathbf{c}-e_{q}$ with $q \in R$ are the end vertices $q=r_{i-1}$ and $q=r_{i}$ while the in between vertices $\mathbf{c}-e_{q}$ all have $q \in Q$. We claim that each edge from $\mathbf{c}-e_{r_{i-1}}$ to $\mathbf{c}-e_{r_{i}}$ is an $R$-linear syzygy edge. This will prove the lemma.

Let the path from $\mathbf{c}-r_{i-1}$ to $\mathbf{c}-r_{i}$ be

$$
\mathbf{c}-e_{r_{i-1}}=\mathbf{c}-e_{q_{0}}, \mathbf{c}-e_{q_{1}}, \ldots, \mathbf{c}-e_{q_{t}}=\mathbf{c}-e_{r_{i}}
$$


where $q_{1}, \ldots, q_{t-1}$ are all in $Q$. We must show that

$$
X_{p}\left(\mathbf{c}-e_{r_{i-1}}\right)=X_{p}\left(\mathbf{c}-e_{r_{i}}\right) \text { for } p \in R \backslash\left\{r_{i-1}, r_{i}\right\} .
$$

But since the edges on the path are linear syzygy edges we have

$$
X_{p}\left(\mathbf{c}-e_{q_{j-1}}\right)=X_{p}\left(\mathbf{c}-e_{q_{j}}\right) \text { for } p \in \operatorname{supp}(\mathbf{c}) \backslash\left\{q_{j-1}, q_{j}\right\} .
$$

Since $q_{1}, \ldots, q_{t-1}$ are not in $R$ we get (11)

Given two $m$-tuples $\mathbf{a}=\left(a_{1}, \ldots, a_{m}\right)$ and $\mathbf{b}=\left(b_{1}, \ldots, b_{m}\right)$ in $\Delta_{m}(n)$. Let $[m]=A \cup B$ be the disjoint set partition such that $a_{i} \geq b_{i}$ for $i \in A$ and $a_{i}<b_{i}$ for $i \in B$. We let

$$
d(\mathbf{a}, \mathbf{b})=\sum_{i \in B}\left(b_{i}-a_{i}\right)=\sum_{i \in A}\left(a_{i}-b_{i}\right)
$$

be a measure for the distance between $\mathbf{a}$ and $\mathbf{b}$. Note that the distance may be measured using only the index set $B$ which in turn depends on the ordered set $(\mathbf{a}, \mathbf{b})$. It should thus really be written $B(\mathbf{a}, \mathbf{b})$. When we measure the distance between two vertices, the first will normally be denoted by a variation on $\mathbf{a}$ and the second a variation on $\mathbf{b}$.

We have the partial order $\mathbf{a} \leq \mathbf{b}$ if each $a_{i} \leq b_{i}$. The least upper bound for $\mathbf{a}$ and $\mathbf{b}$ in this partial order is

$$
\mathbf{a} \vee \mathbf{b}=\left(\max \left\{a_{1}, b_{1}\right\}, \ldots, \max \left\{a_{m}, b_{m}\right\}\right) .
$$

Recall that $m(\mathbf{a})$ and $m(\mathbf{b})$ are the monomials in positions $\mathbf{a}$ and $\mathbf{b}$ respectively. The following is the main and crucial ingredient on the proof of Theorem 4.5.

Proposition 4.11. Given $\mathbf{a}, \mathbf{b} \in \Delta_{m}(n)$. Suppose for every $\mathbf{c} \in \Delta_{m}(n+1)$ the linear syzygy edges $\mathrm{LS}(\mathbf{c})$ contains a spanning tree for the down-graph $D(\mathbf{c})$. Then there is a path

in $\Delta_{m}(n)$ such that:

$$
\mathbf{a}=\mathbf{b}_{0}, \mathbf{b}_{1}, \ldots, \mathbf{b}_{N}=\mathbf{b}
$$

(1) Every $\mathbf{b}_{i} \leq \mathbf{a} \vee \mathbf{b}$,

(2) Every $m\left(\mathbf{b}_{i}\right)$ divides the least common multiple $\operatorname{lcm}(m(\mathbf{a}), m(\mathbf{b}))$,

(3) The edge from $\mathbf{b}_{i-1}$ to $\mathbf{b}_{i}$ is a linear syzygy edge for each $i$.

We call such a path an LS-path from $\mathbf{a}$ to $\mathbf{b}$.

We first show this when the distance between $\mathbf{a}$ and $\mathbf{b}$ is one.

Lemma 4.12. When the distance between $\mathbf{a}$ and $\mathbf{b}$ is one, there is an LS-path from $\mathbf{a}$ to $\mathbf{b}$.

Proof. In this case there is a unique $\mathbf{c} \in \Delta_{m}(n+1)$ such that $\mathbf{a}=\mathbf{c}-e_{i}$ and $\mathbf{b}=\mathbf{c}-e_{j}$, and then $\mathbf{a} \vee \mathbf{b}=\mathbf{c}$. Let $T$ in the linear syzygy edges $\operatorname{LS}(\mathbf{c})$ be a spanning tree for $D(\mathbf{c})$. Then there is a unique path from $\mathbf{a}$ to $\mathbf{b}$ in $T$. We show that for any $m(\mathbf{u})$ on this path, $m(\mathbf{u})$ divides $\operatorname{lcm}(m(\mathbf{a}), m(\mathbf{b}))$. It is enough to show for any $k \in \operatorname{supp}(\mathbf{c})$ that any $x_{k}$-variable in $m(\mathbf{u})$ is contained in either the $x_{k}$-variables of $m(\mathbf{a})$ or the $x_{k}$-variables of $m(\mathbf{b})$.

Case 1. If the path from $\mathbf{a}$ to $\mathbf{b}$ does not contain $\mathbf{c}-e_{k}$, then since all edges on the path are linear syzygy edges, $X_{k}\left(\mathbf{b}_{i-1}\right)=X_{k}\left(\mathbf{b}_{i}\right)$ for every $i$.

Case 2. It the path from $\mathbf{a}$ to $\mathbf{b}$ contains $\mathbf{c}-e_{k}$, say this is $\mathbf{b}_{t}$, then: 
- $X_{k}\left(\mathbf{b}_{i-1}\right)=X_{k}\left(\mathbf{b}_{i}\right)$ for $i<t$ and these are all equal to $X_{k}(\mathbf{a})$,

- $X_{k}\left(\mathbf{b}_{i}\right)=X_{k}\left(\mathbf{b}_{i+1}\right)$ for $i>t$ and these are all equal to $X_{k}(\mathbf{b})$,

- $X_{k}\left(\mathbf{b}_{t}\right)=X_{k}\left(\mathbf{c}-e_{k}\right)$ is contained in both $X_{k}(\mathbf{a})$ and $X_{k}(\mathbf{b})$ by the isotonicity of $X_{k}$.

Proof of Proposition 4.11. We do this in three parts.

Part A. In this part we define the setting. Take distinct $\mathbf{a}$ and $\mathbf{b}$ in $\Delta_{m}(n)$. Assume the distance $d(\mathbf{a}, \mathbf{b}) \geq 2$ since the case of distance 1 is done above. Let

$$
B=B(\mathbf{a}, \mathbf{b})=\left\{i \mid b_{i}>a_{i}\right\}, \quad A_{>}=\left\{i \mid a_{i}>b_{i}\right\}, \quad A_{=}=\left\{i \mid a_{i}=b_{i}\right\},
$$

and $A=A_{>} \cup A_{=}$. We want to consider $\mathbf{b}^{\prime}$ which are in some sense "close" to $\mathbf{b}$. Let $P(\mathbf{b})$ consist of all $\mathbf{b}^{\prime} \in \Delta_{m}(n)$ such that

1. - For $i$ in $B$, $\mathbf{b}$ and $\mathbf{b}^{\prime}$ have equal $i$ 'th coordinate.

- For $i$ in $A, b_{i}^{\prime} \leq a_{i}$.

2. There is some LS-path from $\mathbf{b}^{\prime}$ to $\mathbf{b}$ where the vertices $\mathbf{u}$ on the path satisfy

$-\mathbf{u} \leq \mathbf{a} \vee \mathbf{b}$ (which since we are assuming an LS-path, follows from 1 above),

- $m(\mathbf{u})$ divides $\operatorname{lcm}(m(\mathbf{a}), m(\mathbf{b}))$.

Now let the subset $A_{1}$ of $A$ consist of all coordinate indices $i$ in $A$ such that there is some $\mathbf{b}^{\prime}$ in $P(\mathbf{b})$ with strict inequality $b_{i}^{\prime}<a_{i}$. Let $A_{0}$ be the complement $A \backslash A_{1}$. It is the intersection of all the $A_{=}$associated to $\mathbf{b}^{\prime}$ in $P(\mathbf{b})$. In particular note i) that $A_{1} \supseteq A_{>}$(since $\left.\mathbf{b} \in P(\mathbf{b})\right)$. So $A_{1}$ is not empty and $A_{0} \subseteq A_{=}$.

$$
\text { ii) } b_{i}^{\prime}=a_{i}=b_{i} \text { for } i \in A_{0}, \quad \text { iii) } d\left(\mathbf{a}, \mathbf{b}^{\prime}\right)=d(\mathbf{a}, \mathbf{b}) \text { for } \mathbf{b}^{\prime} \in P(\mathbf{b}) \text {, }
$$

the latter because the $B$-sets $B\left(\mathbf{a}, \mathbf{b}^{\prime}\right)=B(\mathbf{a}, \mathbf{b})$ and the distance may be measured by this, confer (12).

Choose $\beta \in B$ and let $R=A_{1} \cup\{\beta\}$. Consider the down-graph $D_{R}\left(\mathbf{a}+e_{\beta}\right)$. With $\beta$ fixed, there is by Lemma 4.9 an $R$-linear syzygy edge $\left(\mathbf{a}+e_{\beta} ; \beta, \alpha\right)$ for some $\alpha$ in $A_{1}$. This is an edge from $\mathbf{a}$ to $\mathbf{a}+e_{\beta}-e_{\alpha}$. Since $\alpha \in A_{1}$ there is a $\mathbf{b}^{\prime} \in P(\mathbf{b})$ with $b_{\alpha}^{\prime}<a_{\alpha}$. Then the $B$-sets (see (12) $) B\left(\mathbf{a}+e_{\beta}-e_{\alpha}, \mathbf{b}^{\prime}\right) \subseteq B\left(\mathbf{a}, \mathbf{b}^{\prime}\right)$, with equality unless $a_{\beta}+1=b_{\beta}$ in which case the the former set comes from removing $\beta$ from the latter. In any case the distances

$$
d\left(\mathbf{a}+e_{\beta}-e_{\alpha}, \mathbf{b}^{\prime}\right)=d\left(\mathbf{a}, \mathbf{b}^{\prime}\right)-1(=d(\mathbf{a}, \mathbf{b})-1) .
$$

By induction on distance there is an LS-path

$$
\mathbf{a}+e_{\beta}-e_{\alpha}=\mathbf{b}^{0}, \mathbf{b}^{1}, \cdots, \mathbf{b}^{N}=\mathbf{b}^{\prime} .
$$

So we have

- each $m\left(\mathbf{b}^{j}\right)$ divides $\operatorname{lcm}\left(m\left(\mathbf{a}+e_{\beta}-e_{\alpha}\right), m\left(\mathbf{b}^{\prime}\right)\right)$,

- each $\mathbf{b}^{j} \leq\left(\mathbf{a}+e_{\beta}-e_{\alpha}\right) \vee \mathbf{b}^{\prime} \leq \mathbf{a} \vee \mathbf{b}$.

There may be elements on this LS-path such that $m\left(\mathbf{b}^{j}\right)$ does not divide $\operatorname{lcm}(m(\mathbf{a}), m(\mathbf{b}))$. But $m\left(\mathbf{b}^{j}\right)$ will divide if we get sufficiently close to $\mathbf{b}^{\prime}$ as we show in Fact 4.14 below. If $\mathbf{a}+e_{\beta}-e_{\alpha}$ and $\mathbf{b}^{\prime}$ have equal $i^{\prime}$ th coordinates for every $i \in B$, the distance $d(\mathbf{a}, \mathbf{b})$ would be 1 , but we are assuming the distance is $\geq 2$. So $\mathbf{a}+e_{\beta}-e_{\alpha}$ and $\mathbf{b}^{\prime}$ do not have 
equal $i$ 'th coordinate for every $i \in B$. Let $\mathbf{b}^{p}$ be the last element on the path (14) for which $b_{k}^{p} \neq b_{k}^{\prime}$ for some $k \in A_{0} \cup B$.

Part B. In this part, in Facts 4.13, 4.14, and 4.15, we investigate in detail the path from $\mathbf{b}^{p}$ to $\mathbf{b}^{\prime}=\mathbf{b}^{N}$.

Fact 4.13. There is a unique $k \in A_{0} \cup B$ such that $b_{k}^{p} \neq b_{k}^{\prime}$. For every $i \in A_{0} \cup B$ and $j=p, \ldots, N$ we have $b_{i}^{j}=b_{i}^{\prime}=b_{i}$, save for $j=p$ and $i=k$ when $b_{k}^{p}=b_{k}^{p+1}-1$ which is $b_{k}^{\prime}-1=b_{k}-1$.

Proof. Clearly, by the definition of $\mathbf{b}^{p}$, we have that the $b_{i}^{j}$ are all equal to $b_{i}^{\prime}$ for $i \in A_{0} \cup B$ and $j=p+1, \ldots, N$. If $i \in B$ then $b_{i}^{\prime}=b_{i}$ since $\mathbf{b}^{\prime} \in P(\mathbf{b})$. If $i \in A_{0}$ then $b_{i}^{\prime}=b_{i}$ by (13). Furthermore we must have $b_{k}^{p}=b_{k}^{p+1} \pm 1$ which is $b_{k}^{\prime} \pm 1$. Note that

$$
b_{i}^{p} \leq \max \left\{a_{i}, b_{i}\right\}=b_{i}, \quad i \in A_{0} \cup B .
$$

Since the edge from $\mathbf{b}^{p}$ to $\mathbf{b}^{p+1}$ is an LS-edge, there are exactly two coordinates $k, \ell$ where $\mathbf{b}^{p}$ and $\mathbf{b}^{p+1}$ are distinct. Since $b_{k}^{p+1}=b_{k}$ we must by (15) have $b_{k}^{p}=b_{k}^{p+1}-1$. Then we will have $b_{\ell}^{p}=b_{\ell}^{p+1}+1$. If $\ell \in A_{0} \cup B$ then $b_{\ell}^{p+1}=b_{\ell}^{\prime}=b_{\ell}$ which together with (15) gives a contradiction. Thus we have a unique $k$ in $A_{0} \cup B$. Whence when $i \in A_{0} \cup B$ and $i \neq k$ we have $b_{i}^{p}=b_{i}^{p+1}=b_{i}^{\prime}=b_{i}$.

Fact 4.14. For all $\mathbf{b}^{j}$ with $j=p, \ldots, N$ we have

i) $\mathbf{b}^{j} \leq \mathbf{a} \vee \mathbf{b}$

ii) $m\left(\mathbf{b}^{j}\right)$ divides $\operatorname{lcm}(m(\mathbf{a}), m(\mathbf{b}))$.

Proof. Part i) is already noted when we defined the path from $\mathbf{a}+e_{\beta}-e_{\alpha}$ to $\mathbf{b}^{\prime}$. Now we do part ii). We know that $m_{t}\left(\mathbf{b}^{j}\right)$ divides $\operatorname{lcm}\left(m_{t}\left(\mathbf{a}+e_{\beta}-e_{\alpha}\right), m_{t}\left(\mathbf{b}^{\prime}\right)\right)$ for every $t$.

a. Let $t \in A_{1}$. There is an $R=A_{1} \cup\{\beta\}$-linear syzygy between $m(\mathbf{a})$ and $m\left(\mathbf{a}+e_{\beta}-e_{\alpha}\right)$ and so $m_{t}(\mathbf{a})=m_{t}\left(\mathbf{a}+e_{\beta}-e_{\alpha}\right)$ for $t \in A_{1} \backslash\{\alpha\}$. For $t=\alpha$ then $m_{\alpha}\left(\mathbf{a}+e_{\beta}-e_{\alpha}\right)$ divides $m_{\alpha}(\mathbf{a})$ by $X_{\alpha}$ being isotone. From this and the defining requirements on $\mathbf{b}^{\prime}$, it follows that $m_{t}\left(\mathbf{b}^{j}\right)$ divides $\operatorname{lcm}(m(\mathbf{a}), m(\mathbf{b}))$ for $t \in A_{1}$.

b. Let now $t \in A_{0} \cup B$. The edges on the path between $\mathbf{b}^{p}$ and $\mathbf{b}^{\prime}$ are LS-edges. It follows then from Fact 4.13 that for each $t \in\left(A_{0} \cup B\right) \backslash\{k\}$ and $j=p, \ldots, N$ that $m_{t}\left(\mathbf{b}^{j}\right)=m_{t}\left(\mathbf{b}^{\prime}\right)$. When $t=k$, since $b_{k}^{p}=b_{k}^{p+1}-1$ the part $m_{k}\left(\mathbf{b}^{p}\right)$ divides $m_{k}\left(\mathbf{b}^{p+1}\right)$ and we will further have all $m_{k}\left(\mathbf{b}^{j}\right)$ equal for $j=p+1, \ldots, N$, since these $\mathbf{b}^{j}$ are related by LS-edges, and have the same $k^{\prime}$ th coordinate. The upshot is that also $m_{k}\left(\mathbf{b}^{j}\right)$ divides $m_{k}\left(\mathbf{b}^{\prime}\right)$. Thus $m_{t}\left(\mathbf{b}^{j}\right)$ divides $m_{t}\left(\mathbf{b}^{\prime}\right)$ for every $t \in A_{0} \cup B$. Since $\mathbf{b}^{\prime} \in P(\mathbf{b})$, the $m_{t}\left(\mathbf{b}^{\prime}\right)$ divide $\operatorname{lcm}(m(\mathbf{a}), m(\mathbf{b}))$ and we are done.

The following is the main technical detail that makes the proof work. It ensures that we can use induction on distance in Part $\mathrm{C}$. To achieve this we need $A_{0}$ as small as possible, and therefore introduced the neighbourhood $P(\mathbf{b})$ of $\mathbf{b}$. (But this had to be balanced against $A_{1}$, the complement of $A_{0}$ in $A$, not being too big in order to construct the LS-path in (14) by induction.)

Fact 4.15. The coordinate $k \in B$.

Proof. By Fact $4.14 m\left(\mathbf{b}^{j}\right)$ divides $\operatorname{lcm}(m(\mathbf{a}), m(\mathbf{b}))$ and $\mathbf{b}^{j} \leq \mathbf{a} \vee \mathbf{b}$ for $j=p, \ldots, N$. If $k \in A_{0}$ then by Fact $4.13 b_{i}^{p}=b_{i}^{\prime}=b_{i}$ for each $i \in B$. So $\mathbf{b}^{p}$ fulfills the requirement to be in $P(\mathbf{b})$. But then by (13) $b_{k}^{p}=a_{k}=b_{k}$ contradicting Fact 4.13. 
Part C. In this last part we splice three paths together to make an LS-path from a to b. Consider the distance between $\mathbf{a}$ and $\mathbf{b}^{p}$. For $i \in A$ we have $b_{j}^{p} \leq a_{j}$ since $\mathbf{b}^{p} \leq \mathbf{a} \vee \mathbf{b}$. For $i \in B \backslash\{k\}$ we have $b_{i}^{p}=b_{i}^{\prime}=b_{i}>a_{i}$ and when $i=k$ then $a_{k}<b_{k}$ and $b_{k}^{p}=b_{k}-1$. Thus, by looking at the terms with coordinates in $B$, the distance $d\left(\mathbf{a}, \mathbf{b}^{p}\right)=d(\mathbf{a}, \mathbf{b})-1$. By induction there is an LS-path from $\mathbf{a}$ to $\mathbf{b}^{p}$. We now have three LS-paths which we splice:

- The LS-path from a to $\mathbf{b}^{p}$. All $m(\mathbf{u})$ on this path have i) $\mathbf{u} \leq \mathbf{a} \vee \mathbf{b}^{p} \leq \mathbf{a} \vee \mathbf{b}$ and ii) $m(\mathbf{u})$ divides $\operatorname{lcm}\left(m(\mathbf{a}), m\left(\mathbf{b}^{p}\right)\right)$ which again divides $\operatorname{lcm}(m(\mathbf{a}), m(\mathbf{b}))$ by Fact 4.14 .

- The LS-path from $\mathbf{b}^{p}$ to $\mathbf{b}^{\prime}$. We refer to Fact 4.14 concerning the terms here.

- The LS-path from $\mathbf{b}^{\prime}$ to $\mathbf{b}$ with properties required by the definition of $P(\mathbf{b})$.

Splicing these three LS-paths together we get a path of linear syzygy edges from $m(\mathbf{a})$ to $m(\mathbf{b})$ where all $m(\mathbf{u})$ on this path have i) $\mathbf{u} \leq \mathbf{a} \vee \mathbf{b}$ and ii) $m(\mathbf{u})$ divides $\operatorname{lcm}(m(\mathbf{a}), m(\mathbf{b}))$. Thus we have an LS-path from $\mathbf{a}$ to $\mathbf{b}$.

4.3. Proofs of the main Theorem 4.5. We show first Part a., that if the isotone maps $\left\{X_{i}\right\}$ give a polarization, then for each $\mathbf{c} \in \Delta_{m}(n+1)$ the linear syzygy edges $\mathrm{LS}(\mathbf{c})$ of the down-graph $D(\mathbf{c})$ contain a spanning tree for this down-graph.

Proof of Theorem 4.5, Part a. We assume that the isotone maps $\left\{X_{i}\right\}$ give an ideal $J$ which is a polarization. We shall prove that every down-graph $D(\mathbf{c})$ contains a spanning tree of linear syzygy edges. For simplicity we shall assume supp (c) has full support $[m]=\{1,2, \ldots, m\}$. The arguments work just as well in the general case. Since by Lemma 4.1

$$
\mathbf{m}=\prod_{i=1}^{m} m_{i}\left(\mathbf{c}-e_{i}\right)
$$

of degree $\mathbf{c}-\mathbf{1}$ is a divisor of $m\left(\mathbf{c}-e_{v}\right)$ for any $\mathbf{c}-e_{v}$ in $D(\mathbf{c})$, we may write $m\left(\mathbf{c}-e_{v}\right)=$ $\mathbf{m} \cdot n\left(\mathbf{c}-e_{v}\right)$ where $n\left(\mathbf{c}-e_{v}\right)$ has degree $\mathbf{1}-e_{v}$. For two distinct vertices $\mathbf{c}-e_{v}$ and $\mathbf{c}-e_{w}$ in $D(\mathbf{c})$ we define the distance $d\left(m\left(\mathbf{c}-e_{v}\right), m\left(\mathbf{c}-e_{w}\right)\right)$ to be the number of $k \in[m]$ such that either:

- The (unique) $x_{k}$-variables of $n\left(\mathbf{c}-e_{v}\right)$ and of $n\left(\mathbf{c}-e_{w}\right)$ are distinct,

- $k=v$ (then $n\left(\mathbf{c}-e_{v}\right)$ has no $x_{v}$-variable),

- $k=w$ (then $n\left(\mathbf{c}-e_{w}\right)$ has no $x_{w}$-variable),

Note that if the distance between $m\left(\mathbf{c}-e_{v}\right)$ and $m\left(\mathbf{c}-e_{w}\right)$ is 2 , then the set of $k$ 's is $\{v, w\}$ and there is a linear syzygy between these monomials. Suppose now the vertices of $D(\mathbf{c})$ can be divided into two distinct subsets $V_{1}$ and $V_{2}$ such that there is no linear syzygy edge between a vertex in $V_{1}$ and a vertex in $V_{2}$.

Let $\mathbf{c}-e_{v}$ in $V_{1}$ and $\mathbf{c}-e_{w}$ in $V_{2}$ be such that the distance $d$ between $m\left(\mathbf{c}-e_{v}\right)$ and $m\left(\mathbf{c}-e_{w}\right)$ is minimal. We must have $d \geq 3$ and the number of vertices $m \geq 3$. For simplicity we may assume $v=1$ and $w=2$ and that we may write

$$
n\left(\mathbf{c}-e_{2}\right)=x_{1 i_{1}} x_{3 i_{3}} \cdots x_{m i_{m}}, \quad n\left(\mathbf{c}-e_{1}\right)=x_{2 j_{2}} x_{3 j_{3}} \cdots x_{m j_{m}},
$$

where $x_{p i_{p}} \neq x_{p j_{p}}$ for $p=3, \ldots, d$ and $x_{p i_{p}}=x_{p j_{p}}$ for $p>d$ where $d \geq 3$. 
Consider the graded ring $k\left[\check{X}_{1}, \ldots, \check{X}_{m}\right] / J$ and divide out by the regular sequence $x_{p i_{p}}-x_{p j_{p}}$ for $p=4, \ldots, d$. This is a regular sequence, since we assume we have a polarization. We get a quotient algebra $k\left[\check{X}_{1}^{\prime}, \ldots, \check{X}_{m}^{\prime}\right] / J^{\prime}$ and denote by $x_{p}$ the class $\overline{x_{p i_{p}}}=\overline{x_{p j_{p}}}$ for $p \geq 4$. In $J^{\prime}$ we have generators

$$
\begin{array}{ll}
\bar{m}\left(\mathbf{c}-e_{2}\right)=\overline{\mathbf{m}} \cdot \bar{n}\left(\mathbf{c}-e_{2}\right), & \bar{n}\left(\mathbf{c}-e_{2}\right)=x_{1 i_{1}} x_{3 i_{3}} x_{4} \cdots x_{m} \\
\bar{m}\left(\mathbf{c}-e_{1}\right)=\overline{\mathbf{m}} \cdot \bar{n}\left(\mathbf{c}-e_{1}\right), & \bar{n}\left(\mathbf{c}-e_{1}\right)=x_{2 j_{2}} x_{3 j_{3}} x_{4} \cdots x_{m}
\end{array}
$$

Now $x_{3 i_{3}}-x_{3 j_{3}}$ is a non-zero divisor of $k\left[\check{X}_{1}^{\prime}, \cdots, \check{X}_{m}^{\prime}\right] / J^{\prime}$. Consider

$$
\left(x_{3 i_{3}}-x_{3 j_{3}}\right) x_{1 i_{1}} x_{2 j_{2}} x_{4} \cdots x_{m} \cdot \overline{\mathbf{m}} \text {. }
$$

It is zero in this quotient ring, and so

$$
\overline{\mathbf{m}^{\prime}}=x_{1 i_{1}} x_{2 j_{2}} x_{4} \cdots x_{m} \cdot \overline{\mathbf{m}}
$$

is zero in this quotient ring and so must be a generator of $J^{\prime}$ of degree $\mathbf{c}-e_{3}$. But then the generator of this degree in the polarization $J$ must be

$$
\mathbf{m}^{\prime}=x_{1 i_{1}} x_{2 j_{2}} x_{4 k_{4}} \cdots x_{m k_{m}} \cdot \mathbf{m}
$$

where each $k_{p}$ is either $i_{p}$ or $j_{p}$. Hence all $k_{p}=i_{p}=j_{p}$ for $p>d$. But then we see that the distance between $\mathbf{m}^{\prime}$ and $m\left(\mathbf{c}-e_{2}\right)$ is $\leq d-1$ and similarly the distance between $\mathbf{m}^{\prime}$ and $m\left(\mathbf{c}-e_{1}\right)$ is $\leq d-1$. Whether $\mathbf{m}^{\prime}$ is now in $V_{1}$ or in $V_{2}$ we see that this contradicts $d$ being the minimal distance.

Proof of Theorem 4.5, Part b. We shall now prove that if each down-graph $D(\mathbf{c})$ contains a spanning tree of linear syzygy edges, then $J$ will be a polarization. Order the variables in each $\check{X}_{i}$ in a sequence $x_{i 1}, x_{i 2}, \ldots, x_{i n}$. Let $\check{X}_{i}^{\prime}$ consist of $x_{i 1}, \ldots, x_{i p_{i}}, x_{i}$ so we have a surjection $\check{X}_{i} \rightarrow \check{X}_{i}^{\prime}$ for each $i$ sending $x_{i j}$ to itself for $j \leq p_{i}$, and to $x_{i}$ for $j>p_{i}$. Denote the image of $J$ in $k\left[\check{X}_{1}^{\prime}, \ldots, \check{X}_{m}^{\prime}\right]$ by $J^{\prime}$ and the image of $m(\mathbf{a})$ by $m^{\prime}(\mathbf{a})$. The quotient ring $k\left[\check{X}_{1}^{\prime}, \ldots, \check{X}_{m}^{\prime}\right] / J^{\prime}$ is obtained from $k\left[\check{X}_{1}, \ldots \check{X}_{m}\right] / J$ by dividing out by variable differences $x_{i j}-x_{i, j+1}$ for $i=1, \ldots m$ and $j>p_{i}$. We assume this is a regular sequence. We show that if we now divide out by $x_{i, p_{i}}-x_{i, p_{i}+1}$ this is a non-zero divisor of $k\left[\check{X}_{1}^{\prime}, \ldots, \check{X}_{m}^{\prime}\right] / J^{\prime}$. By continuing we get eventually that $k\left[x_{1}, \ldots, x_{m}\right] /\left(x_{1}, \ldots, x_{m}\right)^{n}$ is a regular quotient of $k\left[\check{X}_{1}, \ldots, \check{X}_{m}\right] / J$ and so $J$ is a polarization of $\left(x_{1}, \ldots, x_{m}\right)^{n}$.

We write $x_{i}^{\prime}=x_{i, p_{i}}$. Suppose $\left(x_{i}^{\prime}-x_{i}\right) \cdot \mathbf{f}=0$ in $k\left[\check{X}_{1}^{\prime}, \ldots, \check{X}_{m}^{\prime}\right] / J^{\prime}$ where $\mathbf{f}$ is a polynomial. By Lemma 2.4 (with $x_{i}^{\prime}=x_{i, p_{i}}$ taking the place of $x_{0}$ ), we must show that if $\mathbf{m}$ is a monomial such that $x_{i}^{\prime} \cdot \mathbf{m}=0$ and $x_{i} \cdot \mathbf{m}=0$, then $\mathbf{m}=0$ in the quotient ring. So some generator $m^{\prime}(\mathbf{a})$ of $J^{\prime}$ divides $x_{i}^{\prime} \mathbf{m}$ and some generator $m^{\prime}(\mathbf{b})$ divides $x_{i} \mathbf{m}$.

By Proposition 4.11 there is an LS-path from $m(\mathbf{a})$ to $m(\mathbf{b})$ consisting of linear syzygy edges and such that each $\mathbf{u}$ on this path has $\mathbf{u} \leq \mathbf{a} \vee \mathbf{b}$ and $m(\mathbf{u})$ on this path divides $\operatorname{lcm}(m(\mathbf{a}), m(\mathbf{b}))$. The image $m^{\prime}(\mathbf{u})$ then divides $x_{i}^{\prime} x_{i} \mathbf{m}$. We will show by induction on the length of the path that some monomial $m^{\prime}(\mathbf{u})$ on this path divides $\mathbf{m}$, and so $\mathbf{m}$ is zero in the quotient ring $k\left[\check{X}_{1}^{\prime}, \ldots, \check{X}_{m}^{\prime}\right] / J^{\prime}$.

If the path has length one, there is a linear syzygy edge between $m(\mathbf{a})$ and $m(\mathbf{b})$. Write

$$
x_{i}^{\prime} \cdot \mathbf{m}=m^{\prime}(\mathbf{a}) \cdot n^{0}(\mathbf{a}), \quad x_{i} \cdot \mathbf{m}=m^{\prime}(\mathbf{b}) \cdot n^{0}(\mathbf{b})
$$


Write also $\mathbf{m}=\left(x_{i}^{\prime}\right)^{p}\left(x_{i}\right)^{q} \cdot \mathbf{n}$ where $\mathbf{n}$ does not contain $x_{i}^{\prime}$ or $x_{i}$. If none of $m^{\prime}(\mathbf{a})$ or $m^{\prime}(\mathbf{b})$ divides $\mathbf{m}$, then

$$
m^{\prime}(\mathbf{a})=\left(x_{i}^{\prime}\right)^{p+1}\left(x_{i}\right)^{q^{\prime}} \cdot n^{1}(\mathbf{a}), \quad m^{\prime}(\mathbf{b})=\left(x_{i}^{\prime}\right)^{p^{\prime}}\left(x_{i}\right)^{q+1} \cdot n^{1}(\mathbf{b}),
$$

where $p^{\prime} \leq p$ and $q^{\prime} \leq q$ (and $n^{1}(\mathbf{a})$ and $n^{1}(\mathbf{b})$ do not contain $x_{i}^{\prime}$ or $\left.x_{i}\right)$. But since the edge from $\mathbf{a}$ to $\mathbf{b}$ is a linear syzygy edge, we must have $p^{\prime}=p, q^{\prime}=q$. But a linear syzygy edge involves variables of distinct $x_{i}$-type, which is not so here. Thus one of $m^{\prime}(\mathbf{a})$ or $m^{\prime}(\mathbf{b})$ must divide $\mathbf{m}$.

Suppose now the path has length $\geq 2$.

Case $a_{i} \geq b_{i}$. Let $\mathbf{a}$ to $\mathbf{a}^{\prime}$ be the first edge along the path. Then the coordinate $a_{i}^{\prime} \leq a_{i}$.

If i) the coordinates $a_{i}$ and $a_{i}^{\prime}$ are equal, the $x_{i}$-type variables of $m(\mathbf{a})$ and $m\left(\mathbf{a}^{\prime}\right)$ are the same, since this is a linear syzygy edge. Since $m^{\prime}(\mathbf{a})$ divides $x_{i}^{\prime} \mathbf{m}$ we get that $m_{i}^{\prime}\left(\mathbf{a}^{\prime}\right)$ divides $x_{i}^{\prime} \mathbf{m}$. If ii) $a_{i}^{\prime}<a_{i}$ then when going from $m(\mathbf{a})$ to $m\left(\mathbf{a}^{\prime}\right)$ some $x_{i}$-type variable drops out from $m_{i}(\mathbf{a})$ by isotonicity of $X_{i}$ and so also $m_{i}^{\prime}\left(\mathbf{a}^{\prime}\right)$ divides $x_{i}^{\prime} \mathbf{m}$.

Since the path from $\mathbf{a}$ to $\mathbf{b}$ is an LS-path, $m^{\prime}\left(\mathbf{a}^{\prime}\right)$ divides $x_{i}^{\prime} x_{i} \mathbf{m}$. For $j \neq i$, then $m_{j}^{\prime}\left(\mathbf{a}^{\prime}\right)$ must divide $\mathbf{m}$ since $m_{j}^{\prime}\left(\mathbf{a}^{\prime}\right)$ contains no $x_{i}$-type variable. The upshot is that $m^{\prime}\left(\mathbf{a}^{\prime}\right)$ divides $x_{i}^{\prime} \mathbf{m}$. Considering the LS-path from $\mathbf{a}^{\prime}$ to $\mathbf{b}$, by induction on path length, some $m^{\prime}(\mathbf{u})$ along this path divides $\mathbf{m}$.

Case $a_{i} \leq b_{i}$. Let $\mathbf{b}$ to $\mathbf{b}^{\prime}$ be the first edge along the path going from $\mathbf{b}$ to $\mathbf{a}$. Then the coordinate $b_{i}^{\prime} \leq b_{i}$. Exactly the same argument as in the case above works in this case also.

\section{Three variables Case: Classification of isotone maps}

In the three variables case there is a particular nice classification of the isotone rank preserving maps from $\Delta_{3}(n) \rightarrow B(n)$.

Let $\Delta_{3}(n)$ have the partial order $\geq_{1}$. The symmetric group $S_{n}$ acts on the Boolean poset $B(n)$ and so acts on the set of isotone rank-preserving maps

$$
X: \Delta_{3}(n) \rightarrow B(n) .
$$

Note that for $\mathbf{c} \in \Delta_{3}^{+}(n)$, an edge $(\mathbf{c} ; 2,3)$ being a quadratic syzygy edge, see Example 4.4, is a property invariant under the action of the group $S_{n}$. The following completely describes the combinatorics of such isotone maps. This is quite nice for the three variables case. For the $m$-variables case in general it seems harder to see a nice description.

Proposition 5.1. Let $n \geq 1$. The orbits under the action of $S_{n}$ of rank-preserving isotone maps $X: \Delta_{3}(n) \rightarrow B(n)$ are in one to one correspondence with subsets of $\Delta_{3}^{+}(n+1)$. An isotone map $X$ corresponds to those $\mathbf{c} \in \Delta_{3}^{+}(n+1)$ such that the (c; 2,3$)$-edges of $\Delta_{3}(n)$ are quadratic syzygy edges for $X$.

Since $\Delta_{3}^{+}(n+1)$ is in bijection to $\Delta_{3}(n-2)$ which has cardinality $\left(\begin{array}{l}n \\ 2\end{array}\right)$, the number

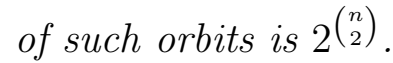

Proof. In each orbit there is exactly one isotone map where the sets

$$
X(0,0, n) \subseteq X(1,0, n-1) \subseteq X(2,0, n-2) \subseteq \cdots \subseteq X(0,0)
$$


are

$$
\emptyset \subseteq\{1\} \subseteq\{1,2\} \subseteq \cdots \subseteq\{1,2, \ldots, n\}
$$

so we need only consider isotone maps which fulfill this condition and show that they are in one-one correspondence with subsets $Q$ of $\Delta_{3}(n-2)$.

Given such an isotone map $X$ we get a subset $Q$ of QS-edges, those $\left(c_{1}, c_{2}, c_{3}\right)$ in $\Delta_{3}^{+}(n+1)$ such that $X\left(c_{1}, c_{2}-1, c_{3}\right) \neq X\left(c_{1}, c_{2}, c_{3}-1\right)$. Conversely given a subset $Q$ of $\Delta_{3}^{+}(n+1)$ we show that this determines uniquely an isotone $X$ fulfilling (16) whose associated set of QS-edges is $Q$. The essential idea is the following simple observation:

Let $A$ be a set of cardinality $p-1$ where $p \geq 1, B, C$ sets containing $A$ of cardinality $p$, and $D$ a set of cardinality $p+1$ containing $B$ and $C$. For fixed $A, B, D$, then either $C=B$ or $C=A \cup(D \backslash B)$. In the latter case note that $A=B \cap C$ and $D=B \cup C$.

Note now first that $X(0, r, n-r)$ is always the empty set. Let $A, B, C, D$ be the four sets of cardinalities respectively $p-1, p, p$ and $p+1$ :

$$
X(p-1,1, n-p), X(p, 1, n-p-1), X(p, 0, n-p), X(p+1,0, n-p-1) .
$$

The last two are known by (16). By induction on $p$ the first is known. Then the second is completely determined by whether the edge $((p, 1, n-p) ; 2,3)$ is a LS- or QS-edge. Thus given $Q$ we may determine all the $X(p, 1, n-p-1)$ for $p=1, \ldots, n-1$. Then we may continue and similarly determine all $X(p, 2, n-p-2)$ for $p=1, \ldots, n-2$ and so on.

\section{Degree two Case: Polarizations of $\left(x_{1}, x_{2}, \ldots, x_{m}\right)^{2}$}

We show that polarizations of the second power $\left(x_{1}, x_{2}, \ldots, x_{m}\right)^{2}$ of the maximal ideal are in one-to-one correspondence with oriented trees with edges labels $1,2, \ldots, m$. Moreover we show that the isomorphism classes of polarizations are in one-to-one correspondence with trees on $(m+1)$ vertices.

First we recall a construction given in [10]. Given a directed tree $T$ (the edges are directed) with edges labelled $1,2, \ldots, m$. The label of an edge $e$ is denoted $l(e)$ Let $\check{X}_{i}=\left\{x_{i 0}, x_{i 1}\right\}$ for $i=1, \ldots, m$. We construct a monomial ideal $K(T)$ in $k\left[\check{X}_{1}, \ldots, \check{X}_{m}\right]$ generated by $(m+1)$ monomials, one for each vertex of $T$. Consider a vertex $v$ of the tree $T$. If an edge $e$ in the tree $T$ is pointing in the direction towards $v$ (this does not meant that $e$ and $v$ have to be incident) let $e_{t o}(v)=1$ and if $e$ is pointing in the opposite direction, let $e_{t o}(v)=0$. To the vertex $v$ of the tree $T$ associate a monomial $m_{v}$ of degree $m$

$$
m_{v}=\prod_{e \in T} x_{l(e), e_{t o}(v)},
$$

and let $I(T)$ be the ideal in $k\left[\check{X}_{1}, \ldots, \check{X}_{m}\right]$ generated by these monomials. We see that the $m_{v}$ are rainbow monomials. By [10] this is a Cohen-Macaulay monomial ideal of codimension two with $m$-linear resolution.

We now construct another monomial ideal $J(T)$ in this polynomial ring as follows. For each pair of distinct vertices $v, w$ of $T$ there is a unique path (forgetting the direction 

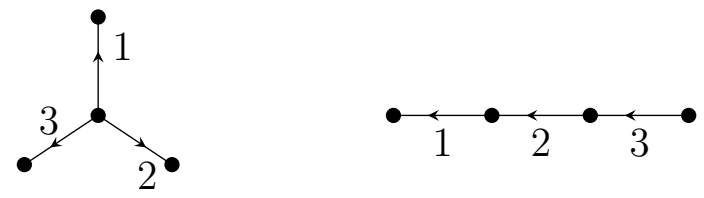

FiguRE 6.
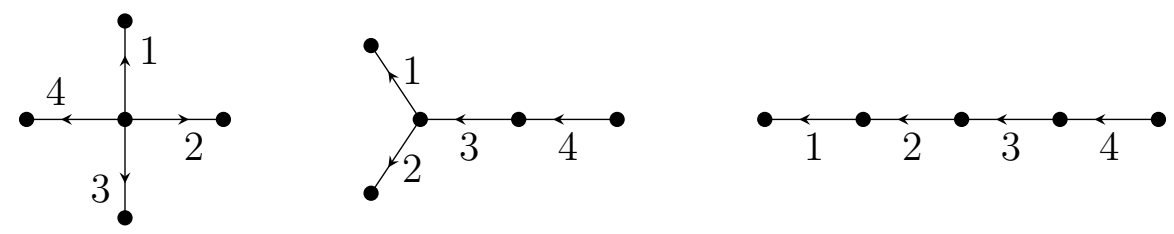

FiguRE 7.

of the edges) between $v$ and $w$. Let $e$ be the edge on this path incident to $v$ and $f$ the edge on this path incident to $w$. Define the monomial $m_{v, w}$ of degree two

$$
m_{v, w}=x_{l(e), e_{t o}(w)} x_{l(f), f_{t o}(v)},
$$

and let $J(T)$ be the ideal generated by all these monomials.

Theorem 6.1. Let $T$ be a directed tree with edges labelled by $1,2, \ldots, m$.

a. The ideals $J(T)$ and $I(T)$ are Alexander duals.

b. The ideal $J(T)$ is a polarization of $\left(x_{1}, \ldots, x_{m}\right)^{2}$, and every polarization of the latter ideal is isomorphic as a monomial ideal to some $J(T)$.

c. Two polarizations $J(T)$ and $J\left(T^{\prime}\right)$ are isomorphic as monomial ideals if and only if the underlying (unlabelled, undirected) trees of $T$ and $T^{\prime}$ are isomorphic.

Example 6.2. The two trees in Figure6 give the non-isomorphic polarizations of $\left(x_{1}, x_{2}, x_{3}\right)^{2}$. The first tree gives the standard polarization

$$
\left(x_{11} x_{12}, x_{11} x_{21}, x_{21} x_{22}, x_{11} x_{31}, x_{21} x_{31}, x_{31} x_{32}\right) .
$$

The second tree gives the b-polarization

$$
\left(x_{11} x_{12}, x_{11} x_{22}, x_{21} x_{22}, x_{11} x_{32}, x_{21} x_{32}, x_{31} x_{32}\right),
$$

which is the letterplace ideal $L(2,3)$ of [13].

Example 6.3. The trees with five vertices, Figure 7, decorated with direction and labelling, give the three non-isomorphic polarizations of $\left(x_{1}, x_{2}, x_{3}, x_{4}\right)^{2}$.

The first tree gives the standard polarization. The second tree gives the polarization:

$$
\left(x_{11} x_{12}, x_{21} x_{22}, x_{31} x_{32}, x_{41} x_{42}, x_{11} x_{21}, x_{11} x_{32}, x_{11} x_{42}, x_{21} x_{32}, x_{21} x_{42}, x_{31} x_{42}\right)
$$

The third tree gives the b-polarization:

$$
\left(x_{11} x_{12}, x_{21} x_{22}, x_{31} x_{32}, x_{41} x_{42}, x_{11} x_{22}, x_{11} x_{32}, x_{11} x_{42}, x_{21} x_{32}, x_{21} x_{42}, x_{31} x_{42}\right),
$$

which is the letterplace ideal $L(2,4)$. 
Proof of Theorem 6.1. a. Consider the generator $m_{v, w}=x_{l(e), e_{t o}(w)} x_{l(f), f_{t o}(v)}$ of $J(T)$. Recall that $e$ is incident to $v$. Let $u$ be another vertex of $T$. If the first variable of $m_{v, w}$ is not in the monomial $m_{u}$ then $w$ and $u$ are in distinct directions from $v$. If the second variable is not in $m_{u}$, then $v$ and $u$ are in distinct directions from $w$, but these two situations together are not possible in a tree.

Hence all the $\left(\begin{array}{c}m+1 \\ 2\end{array}\right)$ monomials $m_{v, w}$ are in the Alexander dual of $I(T)$. But $I(T)$ has $m$-linear resolution and is Cohen-Macaulay of codimension two by [10]. Such an ideal is easily seen to have multiplicity $\left(\begin{array}{c}m+1 \\ 2\end{array}\right)$. For a squarefree monomial ideal $I$ in a polynomial ring $S$, the multiplicity of $I$ (or really of $S / I$ ) equals the number of the facets of maximal dimension of $\Delta(I)$, see Section 6.11 and equation (6.4) in [17]. Since $I(T)$ is Cohen-Macaulay all of its facets have the same dimension. So there are $\left(\begin{array}{c}m+1 \\ 2\end{array}\right)$ facets. Since $I(T)$ has codimension two the facets have cardinality $2 m-2$. The Alexander dual of $I(T)$ is then generated by $\left(\begin{array}{c}m+1 \\ 2\end{array}\right)$ quadratic monomials by the standard correspondence between facets of a squarefree monomial ideal and the generators of its Alexander dual, see Lemma 1.5.4 and Corollary 1.5.5 of [17]. So these generators must be precisely the generators of $J(T)$ making it the Alexander dual.

b. The ideal $J(T)$ has codimension $m$, since its Alexander dual $I(T)$ has generators of degree $m$, loc.cit. Dividing out by the variable differences $x_{i 0}-x_{i 1}$ we easily see that we get the ideal $\left(x_{1}, \ldots, x_{m}\right)^{2}$ which also has codimension $m$. Hence this sequence of variable differences is a regular sequence for $k\left[\check{X}_{1}, \ldots, \check{X}_{m}\right] / J(T)$, and so $J(T)$ is a polarization of $\left(x_{1}, \ldots, x_{m}\right)^{2}$.

Conversely let $J$ be a polarization of $\left(x_{1}, \ldots, x_{m}\right)^{2}$. Then $J$ is Cohen-Macaulay of codimension $m$ with 2-linear resolution. The Alexander dual $I$ will then be CohenMacaulay of codimension 2 with $m$-linear resolution, 8]. By [10, Prop.2.4] there is a tree $T$ such that that $I$ is the image of the $I(T)$ by a map of polynomial rings $k\left[\check{X}_{1}, \ldots, \check{X}_{m}\right]$ (the ring where $I(T)$ lives) to $k\left[\check{X}_{1}, \ldots, \check{X}_{m}\right]$ (the ring where $I$ lives), sending the variables in the former ring to monomials in the latter ring. But since the generators of $I$ and $I(T)$ have the same degree, we must map the variables to variables. Hence $I$ is isomorphic to $I(T)$ as a monomial ideal.

c. If the underlying trees are the same then clearly $T$ and $T^{\prime}$ are related by the action of an element of $S_{m} \ltimes\left(\mathbb{Z}_{2}\right)^{m}$, and so also $J(T)$ and $J\left(T^{\prime}\right)$. If the underlying trees are different, then $I(T)$ and $I\left(T^{\prime}\right)$ are not isomorphic: Their linear syzygies are given precisely by the edges of the trees. Any isomorphism between $I(T)$ and $I\left(T^{\prime}\right)$ would induce a bijection of monomial generators, corresponding to a bijection of vertices of the trees, such that the corresponding linear syzygies between generators, would correspond to a bijection between the edges of the trees.

Together with the discussion in Subsection 3.1 the following shows that polarizations of $\left(x_{1}, \ldots, x_{m}\right)^{2}$ define simplicial balls. For the notion of linear quotients consult [17, Section 8.2.1].

Proposition 6.4. The ideal $I(T)$ has linear quotients, and so equivalently ([17, Prop.8.2.5]) the Alexander dual $J(T)$ defines a shellable simplicial complex.

Proof. Let $r$ be any vertex in $T$ and orient all the arrows away from $r$. This gives a partial order on the vertices of the tree. Take a linear extension of this partial order 
and let $I$ be generated by the monomials in an initial segment for this total order. Let $m_{u}$ be the subsequent monomial. We show that $I: m_{u}$ is generated by variables. So let $m \in I: m_{u}$. Then $m \cdot m_{u}$ is divisible by some $m_{v} \in I$. Each of $m_{u}$ and $m_{v}$ have one variable for each edge in $T$. Only the edges on the path between $u$ and $v$ give distinct variables in $m_{u}$ and $m_{v}$. Starting from $u$ let $e$ be the first edge on the path to $v$. Let $w$ be the other end point of $e$. Since $m_{v}$ comes before $m_{u}$ in the total order, we see that $m_{w}$ must also be in the initial segment which generates $I$. Of the variables in $m_{u}$ and $m_{w}$, only the $e$-variable is different. Thus $I: m_{u}$ contains the $e$-variable $x_{l(e), e_{t o}(w)}$ occurring in $m_{w}$. But then this $e$-variable is also in $m_{v}$ and so this variable divides $m$.

\section{Alexander Duals}

Recall that for a squarefree monomial ideal $I$ in a polynomial ring $S$, the Alexander dual ideal $J$ is the monomial ideal in $S$ whose monomials are precisely those that have nontrivial common divisor with every monomial in $I$, or equivalently, every generator of $I$.

We describe the Alexander dual ideal of any polarization of the power $\left(x_{1}, \ldots, x_{m}\right)^{n}$. The description is a direct construction involving the isotone maps $X_{i}, i=1, \ldots, m$. The proof is reduced to study certain baby-versions $\chi_{i}$ of the $X_{i}$ which are isotone maps $\chi_{i}: \Delta_{m}(n) \rightarrow\{0<1\}$.

7.1. Statement and examples. Let $J \subset k\left[\check{X}_{1}, \ldots, \check{X}_{m}\right]$ be a polarization of the ideal $\left(x_{1}, \ldots, x_{m}\right)^{n}$ in $k\left[x_{1}, \ldots, x_{m}\right]$. For any $\mathbf{a} \in \Delta_{m}(n-1)$ we have the up-graph $U(\mathbf{a})$, with vertices $\mathbf{a}+e_{j}$ for $j=1, \ldots, m$. At the vertex $\mathbf{a}+e_{j}$ we have the $x_{j}$-type variables $X_{j}\left(\mathbf{a}+e_{j}\right)$. We take the product of all these variable sets:

$$
\mathcal{M}(\mathbf{a})=\prod_{j=1}^{m} X_{j}\left(\mathbf{a}+e_{j}\right)
$$

It consists of monomials $x_{1 i_{1}} x_{2 i_{2}} \cdots x_{m i_{m}}$ where $x_{j i_{j}}$ is in $X_{j}\left(\mathbf{a}+e_{j}\right)$. Let $I$ be the ideal generated by the monomials in the union of all the $\mathcal{M}(\mathbf{a})$ for $\mathbf{a} \in \Delta_{m}(n-1)$.

Theorem 7.1. The ideal $I$ is the Alexander dual of $J$.

Example 7.2. Consider again the polarization $J$ from Example 4.8. Its graph of linear syzygies is again given in Figure 8, this time labeling each of the up-triangles in the graph with $1, \ldots, 6$. The up-triangle $i$ corresponds to an element $a_{i} \in \Delta_{3}(2)$, where $a_{1}=(2,0,0), a_{2}=(1,1,0), a_{3}=(1,0,1), a_{4}=(0,2,0), a_{5}=(0,1,1)$, and $a_{6}=(0,0,2)$. The sets of monomials $\mathcal{M}\left(\mathbf{a}_{i}\right)$ are: 


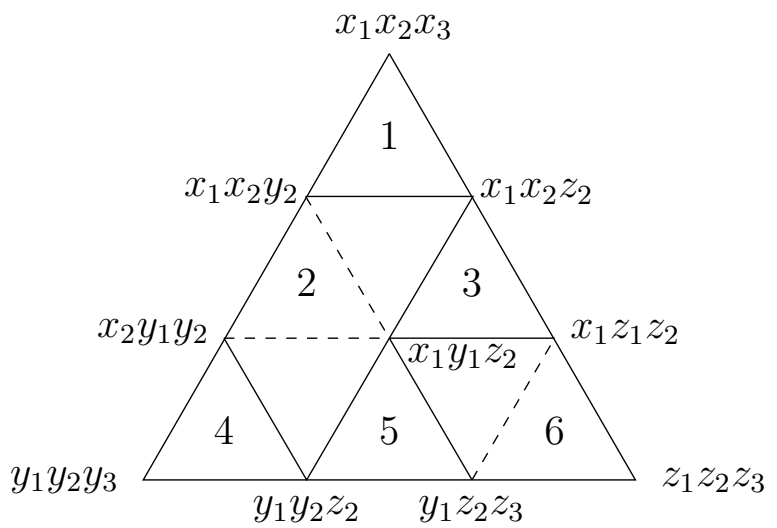

Figure 8.

$$
\begin{aligned}
& \mathcal{M}\left(\mathbf{a}_{1}\right)=\left\{\mathbf{x}_{\mathbf{1}} \mathbf{y}_{\mathbf{2}} \mathbf{z}_{\mathbf{2}}, \mathbf{x}_{\mathbf{2}} \mathbf{y}_{\mathbf{2}} \mathbf{z}_{\mathbf{2}}, \mathbf{x}_{\mathbf{3}} \mathbf{y}_{\mathbf{2}} \mathbf{z}_{\mathbf{2}}\right\} \\
& \mathcal{M}\left(\mathbf{a}_{2}\right)=\left\{\mathbf{x}_{\mathbf{1}} \mathbf{y}_{\mathbf{1}} \mathbf{z}_{\mathbf{2}}, x_{1} y_{2} z_{2}, \mathbf{x}_{\mathbf{2}} \mathbf{y}_{\mathbf{1}} \mathbf{z}_{\mathbf{2}}, x_{2} y_{2} z_{2}\right\} \\
& \mathcal{M}\left(\mathbf{a}_{3}\right)=\left\{\mathbf{x}_{\mathbf{1}} \mathbf{y}_{\mathbf{1}} \mathbf{z}_{\mathbf{1}}, x_{1} y_{1} z_{2}, \mathbf{x}_{\mathbf{2}} \mathbf{y}_{\mathbf{1}} \mathbf{z}_{\mathbf{1}}, x_{2} y_{1} z_{2}\right\} \\
& \mathcal{M}\left(\mathbf{a}_{4}\right)=\left\{x_{2} y_{1} z_{2}, x_{2} y_{2} z_{2}, \mathbf{x}_{\mathbf{2}} \mathbf{y}_{\mathbf{3}} \mathbf{z}_{\mathbf{2}}\right\} \\
& \mathcal{M}\left(\mathbf{a}_{5}\right)=\left\{x_{1} y_{1} z_{2}, \mathbf{x}_{\mathbf{1}} \mathbf{y}_{\mathbf{1}} \mathbf{z}_{\mathbf{3}}, x_{1} y_{2} z_{2}, \mathbf{x}_{\mathbf{1}} \mathbf{y}_{\mathbf{2}} \mathbf{z}_{\mathbf{3}}\right\} \\
& \mathcal{M}\left(\mathbf{a}_{6}\right)=\left\{x_{1} y_{1} z_{1}, x_{1} y_{1} z_{2}, x_{1} y_{1} z_{3}\right\}
\end{aligned}
$$

The boldface monomials are the ten distinct monomials we find from this process, which in fact generate the Alexander dual $I$ of $J$.

We shall go through several steps in proving the above theorem. It turns out that we will be able to abstract the situation so our arguments will only involve a collection of isotone maps

$$
\chi_{i}: \Delta_{m}(n) \rightarrow\{0<1\}, \quad i=1, \ldots, m
$$

where $\Delta_{m}(n)$ has the partial order $\geq_{i}$, and such that $\chi_{i}(\mathbf{b})=0$ whenever $b_{i}=0$.

Remark 7.3. When $m=3$, it is a curious fact that for each $i$ the number of maps $\chi_{i}$ is the Catalan number $C_{n+1}$ : Such maps are in one-one correspondence with "stacking of coins", [27, Exercise 6.19 hhh].

First we establish some notation. For a monomial $\mathbf{m} \in k\left[\check{X}_{1}, \ldots, \check{X}_{m}\right]$, define maps

$$
\begin{aligned}
\chi_{i, \mathbf{m}}: \Delta_{m}(n) \rightarrow\{0<1\} \\
\quad \mathbf{b} \mapsto \begin{cases}0, & \text { no variable of } X_{i}(\mathbf{b}) \text { is in } \mathbf{m} . \\
1, & \text { some variable of } X_{i}(\mathbf{b}) \text { is in } \mathbf{m} .\end{cases}
\end{aligned}
$$

Note i) this is an isotone map and ii) if $(\mathbf{c} ; j, k)$ is a linear syzygy edge for the isotone maps $\left\{X_{i}\right\}$, then $\chi_{i, \mathbf{m}}\left(\mathbf{c}-e_{j}\right)=\chi_{i, \mathbf{m}}\left(\mathbf{c}-e_{k}\right)$ for every $i \neq j, k$. Note furthermore: 
- That a monomial $\mathbf{m} \in k\left[\check{X}_{1}, \ldots, \check{X}_{m}\right]$ is in the Alexander dual of $J$ means that it has a common variable with every $m(\mathbf{b})$, or for every $\mathbf{b} \in \Delta_{m}(n)$, a common variable with some $m_{i}(\mathbf{b})$ for $i=1, \ldots, m$. This holds if and only if for every such $\mathbf{b}$, there is some $i$ with $\chi_{i, \mathbf{m}}(\mathbf{b})=1$.

- The monomial $\mathbf{m}$ is in $I$ if and only if for some $\mathbf{a} \in \Delta_{m}(n-1)$, it has a common variable with every $X_{j}\left(\mathbf{a}+e_{j}\right)$ for $j=1, \ldots, m$. Thus for such an a we have $\chi_{j, \mathbf{m}}\left(\mathbf{a}+e_{j}\right)=1$ for every $j$.

We now abstract the negations of the above. Consider for $i=1, \ldots, m$ isotone maps as in (17).

Definition 7.4. A multidegree $\mathbf{b} \in \Delta_{m}(n)$ is a full zero point for the collection $\left\{\chi_{i}\right\}$ if $\chi_{i}(\mathbf{b})=0$ for every $i=1, \ldots, m$. An up-simplex $U(\mathbf{a})$ of $\Delta_{m}(n)$ has a zero corner if $\chi_{i}\left(\mathbf{a}+e_{i}\right)=0$ for some $i$.

An edge $(\mathbf{c} ; i, j)$ of $\Delta_{m}(n)$ is a linear syzygy edge for the collection $\left\{\chi_{i}\right\}$ if $\chi_{p}\left(\mathbf{c}-e_{i}\right)=$ $\chi_{p}\left(\mathbf{c}-e_{j}\right)$ for every $p \neq i, j$.

We prove the following.

Theorem 7.5. Given the collection of isotone maps $\left\{\chi_{i}\right\}$ such that for every downgraph of $\Delta_{m}(n)$ the linear syzygy edges for $\left\{\chi_{i}\right\}$ contains a spanning tree. Then $\left\{\chi_{i}\right\}$ has a full zero-point in $\Delta_{m}(n)$ if and only if every up-graph of $\Delta_{m}(n)$ has a zero corner.

As a consequence we get Theorem 7.1.

Proof of Theorem 7.1. That $\mathbf{m} \notin I$ means that every up-graph in $\Delta_{m}(n)$ has a zero

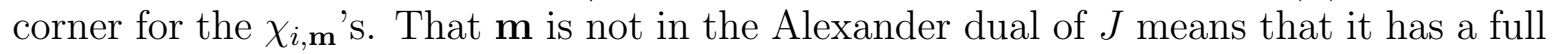
zero-point for the $\chi_{i, \mathbf{m}}$ 's. Hence by Theorem $7.5 I$ will be the Alexander dual of $J$.

7.2. Definitions and key lemma. In order to facilitate our arguments we need to have a more flexible framework to work in. For $\mathbf{d} \in \mathbb{N}^{m}$ with $|\mathbf{d}| \leq n$ and $S \subseteq[m]$, let $\Delta_{S}(n, \mathbf{d})$ be the induced subgraph of $\Delta_{m}(n)$ whose vertices are the degrees $\mathbf{b} \geq \mathbf{d}$ such that $\operatorname{supp}(\mathbf{b}-\mathbf{d}) \subseteq S$. This means:

$$
\text { i) }|\mathbf{b}|=n, \quad \text { ii) } b_{i}=d_{i} \text { for } j \in[m] \backslash S, \quad \text { iii) } b_{i} \geq d_{i} \text { for } i \in S .
$$

(We omit $m$ in $\Delta_{S}(n, \mathbf{d})$ since $m$ is fixed througout.) We normally write $\Delta_{S}(\mathbf{d})$ for $\Delta_{S}(n, \mathbf{d})$ but on a few occasions we want $(n-1)$ or $(n+1)$ instead of $n$ as argument, and use the full notation.

The convex hull of $\Delta_{S}(\mathbf{d})$ in $\mathbb{R}^{S}$ is a simplex of dimension $|S|-1$ if $|\mathbf{d}|<n$. The size of this simplex is $n-|\mathbf{d}|$. For $\mathbf{d}=\mathbf{0}$, the zero degree, we have $\Delta_{[m]}(\mathbf{0})=\Delta_{m}(n)$ as defined earlier. Note that $\Delta_{S^{\prime}}\left(\mathbf{d}^{\prime}\right)$ is a non-empty subset of $\Delta_{S}(\mathbf{d})$ iff:

$$
\text { i) } S^{\prime} \subseteq S, \quad \text { ii) } \mathbf{d}^{\prime} \geq \mathbf{d}, \quad \text { iii) } d_{i}^{\prime}=d_{i} \text { for } i \in[m] \backslash S .
$$

Example 7.6. Let $S=\{2,3,4\}$ and $\mathbf{d}=(1,0,0,0)$. Then $\Delta_{S}(\mathbf{d})$ is the induced subgraph of $\Delta_{4}(3)$ depicted in Figure 9, its convex hull is a simplex of dimension 2, and its size is 2 . If $S^{\prime}=\{2,4\}$, then $\Delta_{S^{\prime}}(\mathbf{d})$ is the subgraph of $\Delta_{S}(\mathbf{d})$ depicted by the thick line in Figure 9, Its convex hull is a simplex of dimension 1, and its size is also 2. 


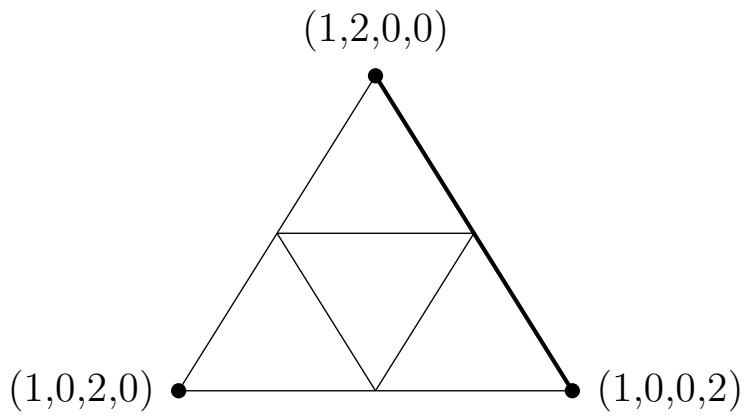

FiguRE 9.

Definition 7.7. For $\mathbf{a} \in \Delta_{S}(n-1, \mathbf{d})$ we get an induced subgraph $U_{S}(\mathbf{a} ; \mathbf{d})$ of $\Delta_{S}(\mathbf{d})$ with vertices $\left\{\mathbf{a}+e_{i} \mid i \in S\right\}$. This is a complete graph on $|S|$ vertices whose convex hull is of dimension $|S|-1$. The graph $U_{S}(\mathbf{a} ; \mathbf{d})$ is an up-graph. The $i \in S$ are the corners of the up-graph.

For $\mathbf{c} \in \Delta_{S}(n+1, \mathbf{d})$ we get an induced subgraph $D_{S}(\mathbf{c} ; \mathbf{d})$ of $\Delta_{S}(\mathbf{d})$ with vertices $\left\{\mathbf{c}-e_{i} \mid i \in \operatorname{supp}(\mathbf{c}-\mathbf{d})\right\}$. This is a complete graph on $|\operatorname{supp}(\mathbf{c}-\mathbf{d})|$ vertices whose convex hull is a simplex of dimension $|\operatorname{supp}(\mathbf{c}-\mathbf{d})|-1$. The graph $D_{S}(\mathbf{c} ; \mathbf{d})$ is a down-graph.

Suppose that for each $j \in S$ we have isotone maps, where we have given $\Delta_{S}\left(\mathbf{d}+e_{j}\right)$ the $\geq_{j}$-ordering:

$$
\chi_{j}: \Delta_{S}\left(\mathbf{d}+e_{j}\right) \rightarrow\{0<1\} .
$$

Note that for $\mathbf{b} \in \Delta_{S}(\mathbf{d})$, the $\chi_{j}(\mathbf{b})$ are defined precisely for $j \in \operatorname{supp}(\mathbf{b}-\mathbf{d})$.

Definition 7.8. A vertex $\mathbf{b} \in \Delta_{S}(\mathbf{d})$ is a full zero point for $\left\{\chi_{i}\right\}_{i \in S}$ if $\chi_{i}(\mathbf{b})=0$ for every $i \in \operatorname{supp}(\mathbf{b}-\mathbf{d})$. An up-graph $U_{S}(\mathbf{a} ; \mathbf{d})$ has a zero corner for $\left\{\chi_{i}\right\}_{i \in S}$ if $\chi_{j}\left(\mathbf{a}+e_{j}\right)=0$ for some $j \in S$.

An edge $(\mathbf{c} ; r, s)$ of $\Delta_{S}(\mathbf{d})$ (note that then $\{r, s\} \subseteq S$ ) is a linear syzygy edge for the $\left\{\chi_{j}\right\}_{j \in S}$ if

$$
\left.\chi_{j}\left(\mathbf{c}-e_{r}\right)\right)=\chi_{j}\left(\mathbf{c}-e_{s}\right), \quad \text { for } j \in \operatorname{supp}(\mathbf{c}-\mathbf{d}) \backslash\{r, s\} .
$$

Lemma 7.9. Suppose we have isotone maps $\left\{\chi_{j}\right\}_{j \in S}$ for $\Delta_{S}(\mathbf{d})$ such that the linear syzygy edges in every down-graph of $\Delta_{S}(\mathbf{d})$ contains a spanning tree.

Let $\Delta_{R}\left(\mathbf{d}^{\prime}\right)$ be a non-empty subgraph of $\Delta_{S}(\mathbf{d})$. For $j \in R$ let $\bar{\chi}_{j}$ be the restriction of $\chi_{j}$ to isotone maps associated to $\Delta_{R}\left(\mathbf{d}^{\prime}\right)$. Then each down-graph of $\Delta_{R}\left(\mathbf{d}^{\prime}\right)$ contains a spanning tree of linear syzygy edges for the $\left\{\bar{\chi}_{j}\right\}_{j \in R}$.

Proof. (This proof is essentially the same as for Lemma 4.9.) Choose a particular down-graph $D_{R}\left(\mathbf{c} ; \mathbf{d}^{\prime}\right)$ of $\Delta_{R}\left(\mathbf{d}^{\prime}\right)$. Let $\mathbf{c}-e_{r}$ and $\mathbf{c}-e_{s}$ be vertices of $D_{R}\left(\mathbf{c} ; \mathbf{d}^{\prime}\right.$ ) (so $\left.r, s \in \operatorname{supp}\left(\mathbf{c}-\mathbf{d}^{\prime}\right)\right)$. We will show that there is a path from $\mathbf{c}-e_{r}$ to $\mathbf{c}-e_{s}$ in the graph $D_{R}\left(\mathbf{c} ; \mathbf{d}^{\prime}\right)$ consisting of linear syzygy edges for the $\left\{\bar{\chi}_{j}\right\}_{j \in R}$.

Since $\mathbf{c} \geq \mathbf{d},|\mathbf{c}|=n+1$ and $c_{i}=d_{i}$ for $i$ outside of $S$, we may consider the down graph $D_{S}(\mathbf{c} ; \mathbf{d})$ in $\Delta_{S}(\mathbf{d})$. We know there is a path in $D_{S}(\mathbf{c} ; \mathbf{d})$ from $\mathbf{c}-e_{r}$ to $\mathbf{c}-e_{s}$ consisting of linear syzygy edges for the $\left\{\chi_{j}\right\}_{j \in S}$. 
It may be broken up into smaller paths: From $\mathbf{c}-e_{r}=\mathbf{c}-e_{r_{0}}$ to $\mathbf{c}-e_{r_{1}}$, from $\mathbf{c}-e_{r_{1}}$ to $\mathbf{c}-e_{r_{2}}, \ldots$, from $\mathbf{c}-e_{r_{p-1}}$ to $\mathbf{c}-e_{r_{p}}=\mathbf{c}-e_{s}$ where on the path from $\mathbf{c}-e_{r_{i-1}}$ to $\mathbf{c}-e_{r_{i}}$ the only vertices $\mathbf{c}-e_{q}$ with $q \in R$ are the end vertices $q=r_{i-1}$ and $q=r_{i}$ while the in between vertices all have $q \in Q=\operatorname{supp}(\mathbf{c}-\mathbf{d}) \backslash R$. We claim that each edge from $\mathbf{c}-e_{r_{i-1}}$ to $\mathbf{c}-e_{r_{i}}$ is a linear syzygy edge for the $\left\{\bar{\chi}_{j}\right\}_{j \in R}$. This will prove the lemma.

Let the path from $\mathbf{c}-e_{r_{i-1}}$ to $\mathbf{c}-e_{r_{i}}$ be

$$
\mathbf{c}-e_{r_{i-1}}=\mathbf{c}-e_{q_{0}}, \mathbf{c}-e_{q_{1}}, \ldots, \mathbf{c}-e_{q_{t-1}}, \mathbf{c}-e_{q_{t}}=\mathbf{c}-r_{i}
$$

where $q_{1}, \ldots, q_{t-1}$ are all in $Q$. We must show that

$$
\bar{\chi}_{p}\left(\mathbf{c}-e_{r_{i-1}}\right)=\bar{\chi}_{p}\left(\mathbf{c}-e_{r_{i}}\right) \text { for } p \in \operatorname{supp}\left(\mathbf{c}-\mathbf{d}^{\prime}\right) \backslash\left\{r_{i-1}, r_{i}\right\} .
$$

But since the edges on the path are linear syzygy edges for the $\left\{\chi_{j}\right\}_{j \in S}$ we have

$$
\chi_{p}\left(\mathbf{c}-e_{q_{j-1}}\right)=\chi_{p}\left(\mathbf{c}-e_{q_{j}}\right) \text { for } p \in \operatorname{supp}(\mathbf{c}-\mathbf{d}) \backslash\left\{q_{j-1}, q_{j}\right\} .
$$

Hence

$$
\chi_{p}\left(\mathbf{c}-e_{r_{i-1}}\right)=\chi_{p}\left(\mathbf{c}-e_{r_{i}}\right) \text { for } p \in \operatorname{supp}(\mathbf{c}-\mathbf{d}) \backslash\left\{r_{i-1}, q_{1}, \ldots, q_{t-1}, r_{i}\right\} .
$$

Since $\operatorname{supp}\left(\mathbf{c}-\mathbf{d}^{\prime}\right) \subseteq R$ and $\left\{q_{1}, \ldots, q_{t-1}\right\} \subseteq S \backslash R$, these are disjoint sets. Also $\operatorname{supp}\left(\mathbf{c}-\mathbf{d}^{\prime}\right) \subseteq \operatorname{supp}(\mathbf{c}-\mathbf{d})$. Hence

$$
\chi_{p}\left(\mathbf{c}-e_{r_{i-1}}\right)=\chi_{p}\left(\mathbf{c}-e_{r_{i}}\right) \text { for } p \in \operatorname{supp}\left(\mathbf{c}-\mathbf{d}^{\prime}\right) \backslash\left\{r_{i-1}, r_{i}\right\} .
$$

This shows (18).

7.3. Full zero point implies a zero corner in every up-graph. We prove this direction of Theorem 7.5 ,

Lemma 7.10. Let $S \subseteq[m]$ have cardinality $\geq 2$. Suppose every down-graph of $\Delta_{S}(\mathbf{d})$ contains a spanning tree of linear syzygies for the isotone maps $\left\{\chi_{j}\right\}_{j \in S}$. Let $\mathbf{b} \in \Delta_{S}(\mathbf{d})$ and $p \in S$. Suppose $\chi_{i}(\mathbf{b})=0$ for every $i \in \operatorname{supp}(\mathbf{b}-\mathbf{d}) \backslash\{p\}$. Then every up-graph $U_{S}(\mathbf{a} ; \mathbf{d})$ in $\Delta_{S}(\mathbf{d})$ with $a_{p} \geq b_{p}$ has a zero corner for the $\left\{\chi_{i}\right\}_{i \in S}$.

Corollary 7.11. Suppose the maps $\left\{\chi_{i}\right\}_{i \in S}$ have a full zero-point in $\Delta_{S}(\mathbf{d})$. Then every up-graph in $\Delta_{S}(\mathbf{d})$ has a zero corner.

Proof Lemma 7.10 and Corollary 7.11. We prove these in tandem by induction on the cardinality $|S|$. We prove Corollary 7.11 by assuming Lemma 7.10, Then we prove Lemma 7.10 by assuming Corollary 7.11 has been proven for all $\Delta_{S^{\prime}}(\mathbf{d})$ when the cardinality $\left|S^{\prime}\right|<|S|$.

Assume we have shown Lemma 7.10, Let $\mathbf{b}$ be a full zero-point for $\Delta_{S}(\mathbf{d})$ and consider an up-graph $U_{S}(\mathbf{a} ; \mathbf{d})$ in $\Delta_{S}(\mathbf{d})$. Since $|\mathbf{b}|=n$ and $|\mathbf{a}|=n-1$, then at least for one $p$ we have $a_{p} \geq b_{p}$. Then Lemma 7.10 implies that $U_{S}(\mathbf{a} ; \mathbf{d})$ is an up-graph with a zero corner, proving Corollary 7.11 .

We now show Lemma 7.10, For simplicity we assume $p=1$. First we do the case $|S|=$ 2 , say $S=\{1,2\}$. Then $\chi_{2}(\mathbf{b})=0$. By isotonicity of $\chi_{2}$ we have $\chi_{2}\left(\mathbf{b}+\lambda e_{1}-\lambda e_{2}\right)=0$ for $\lambda \geq 0$. But letting $\lambda=a_{1}-b_{1}$, the point $\mathbf{b}+\lambda e_{1}-\lambda e_{2}=\mathbf{a}+e_{2}$ is a zero corner for $U_{S}(\mathbf{a} ; \mathbf{d})$. 
Assume now $|S| \geq 3$ and Corollary 7.11 holds for $S^{\prime}$ with $2 \leq\left|S^{\prime}\right|<|S|$. We argue by induction on the difference $a_{1}-b_{1}$.

Case $a_{1}=b_{1}$. Let $S^{\prime}=S \backslash\{1\}$ and $\mathbf{d}^{\prime}=\mathbf{d}+\left(b_{1}-d_{1}\right) e_{1}$. Then $\mathbf{b} \in \Delta_{S^{\prime}}\left(\mathbf{d}^{\prime}\right)$ and $\mathbf{b}$ is a full zero point for the $\left\{\bar{\chi}_{i}\right\}_{i \in S^{\prime}}$. By Corollary 7.11 every up-graph $U_{S^{\prime}}\left(\mathbf{a} ; \mathbf{d}^{\prime}\right)$ in $\Delta_{S^{\prime}}\left(\mathbf{d}^{\prime}\right)$ has a zero corner for $\left\{\bar{\chi}_{i}\right\}_{i \in S^{\prime}}$. Since $\mathbf{a} \in \Delta_{S^{\prime}}\left(n-1, \mathbf{d}^{\prime}\right)$ iff $\mathbf{a} \in \Delta_{S}(n-1, \mathbf{d})$ with $a_{1}=d_{1}^{\prime}=b_{1}$, up-graphs $U_{S^{\prime}}\left(\mathbf{a} ; \mathbf{d}^{\prime}\right)$ in $\Delta_{S^{\prime}}\left(\mathbf{d}^{\prime}\right)$ correspond to up-graphs $U_{S}(\mathbf{a} ; \mathbf{d})$ with $a_{1}=b_{1}$. Then every up-graph $U_{S}(\mathbf{a} ; \mathbf{d})$ with $a_{1}=b_{1}$ has a zero corner for $\left\{\chi_{i}\right\}_{i \in S}$.

Case $a_{1}>b_{1}$. Consider the down-graph $D_{S}\left(\mathbf{b}+e_{1}\right)$. By assumption on the maps $\chi_{i}$, at least one edge $\left(\mathbf{b}+e_{1} ; 1, r\right)$ is a linear syzygy edge, so $\chi_{j}\left(\mathbf{b}+e_{1}-e_{r}\right)=\chi_{j}(\mathbf{b})=0$ for every $j \in \operatorname{supp}(\mathbf{b}-\mathbf{d}) \backslash\{1, r\}$. But $\mathbf{b}+e_{1}-e_{r} \leq_{r} \mathbf{b}$, so $\chi_{r}\left(\mathbf{b}+e_{1}-e_{r}\right) \leq \chi_{p}(\mathbf{b})=0$. Therefore $\chi_{j}\left(\mathbf{b}+e_{1}-e_{r}\right)=0$ for every $j \in \operatorname{supp}(\mathbf{b}-\mathbf{d}) \backslash\{1\}$. Since the difference in first coordinates of $\mathbf{a}$ and $\mathbf{b}+e_{1}-e_{r}$ is one less than $a_{1}-b_{1}$, by induction the up-graph $U_{S}(\mathbf{a} ; \mathbf{d})$ has a zero corner for $\left\{\chi_{j}\right\}_{j \in S}$.

7.4. Every up-graph has a zero corner implies a full zero point. We now prove the other direction of Theorem 7.5. We need the following specific lemma. It says that you can "pull a point" with specific properties in a given direction and into a simplex of smaller size.

Lemma 7.12. Let $\mathbf{b} \in \Delta_{S}(\mathbf{d})$ with $|\mathbf{d}| \leq n-1,1 \in S$, and $b_{1} \leq d_{1}+1$. Suppose

$$
\chi_{i}(\mathbf{b})=0 \text { for } i \in \operatorname{supp}(\mathbf{b}-\mathbf{d}) \backslash\{1\}, \quad \chi_{1}(\mathbf{b})=1 \text { if } 1 \in \operatorname{supp}(\mathbf{b}-\mathbf{d}) .
$$

Let $p \in S \backslash\{1\}$ and $\mathbf{d}^{\prime}=\mathbf{d}+e_{p}$. Then there is some $\mathbf{b}^{\prime} \in \Delta_{S}\left(\mathbf{d}^{\prime}\right)$ with $b_{1}^{\prime} \leq d_{1}^{\prime}+1\left(=d_{1}+1\right)$ such that

$$
\chi_{i}\left(\mathbf{b}^{\prime}\right)=0 \text { for } i \in \operatorname{supp}\left(\mathbf{b}^{\prime}-\mathbf{d}^{\prime}\right) \backslash\{1\}, \quad \chi_{1}\left(\mathbf{b}^{\prime}\right)=1 \text { if } 1 \in \operatorname{supp}\left(\mathbf{b}^{\prime}-\mathbf{d}^{\prime}\right) .
$$

Proof. If $b_{p} \geq d_{p}+1$ we simply let $\mathbf{b}^{\prime}=\mathbf{b}$. So suppose $b_{p}=d_{p}$. Looking at the downgraph $D_{S}\left(\mathbf{b}+e_{p} ; \mathbf{d}\right)$ there is a linear syzygy edge $\left(\mathbf{b}+e_{p} ; p, q\right)$ for some $q \in S \backslash\{p\}$. It goes from $\mathbf{b}$ to $\mathbf{b}^{\prime}=\mathbf{b}+e_{p}-e_{q}$. Let us show that this $\mathbf{b}^{\prime}$ has the desired properties.

We have $\mathbf{b}^{\prime}-\mathbf{d}^{\prime}=\mathbf{b}-\mathbf{d}-e_{q}$ and so $\operatorname{supp}\left(\mathbf{b}^{\prime}-\mathbf{d}^{\prime}\right)$ is $\operatorname{supp}(\mathbf{b}-\mathbf{d})$ with $q$ possibly removed. We see that $p \notin \operatorname{supp}\left(\mathbf{b}^{\prime}-\mathbf{d}^{\prime}\right)$, and so $\chi_{i}\left(\mathbf{b}^{\prime}\right)=\chi_{i}(\mathbf{b})$ for $i \in \operatorname{supp}\left(\mathbf{b}^{\prime}-\mathbf{d}^{\prime}\right) \backslash\{q\}$.

Case $q=1$. In this case $b_{1}^{\prime}=d_{1}$ so $1 \notin \operatorname{supp}\left(\mathbf{b}^{\prime}-\mathbf{d}^{\prime}\right)$ and (19) holds.

Case $q \neq 1$. Note $\mathbf{b}^{\prime} \leq_{q} \mathbf{b}$. So if $q$ is contained in $\operatorname{supp}\left(\mathbf{b}^{\prime}-\mathbf{d}^{\prime}\right)$ then $\chi_{q}\left(\mathbf{b}^{\prime}\right) \leq \chi_{q}(\mathbf{b})=0$. Furthermore $\chi_{1}\left(\mathbf{b}^{\prime}\right)=\chi_{1}(\mathbf{b})=1$ if $1 \in \operatorname{supp}\left(\mathbf{b}^{\prime}-\mathbf{d}^{\prime}\right)$. So again (19) holds.

Proposition 7.13. Let $|\mathbf{d}| \leq n-1$ and $|S| \geq 2$. Suppose every up-graph in $\Delta_{S}(\mathbf{d})$ has a zero corner for the $\left\{\chi_{i}\right\}_{i \in S}$. Then there is an element of $\Delta_{S}(\mathbf{d})$ which is a full zero-point.

Proof. We prove by induction on the size $(n-|\mathbf{d}|)$ and cardinality $|S|$ that the above holds. If $|\mathbf{d}|=n-1$, then $\Delta_{S}(\mathbf{d})$ equals the up-graph $U_{S}(\mathbf{d} ; \mathbf{d})$. If we have a zero at corner $p \in S$, so $\chi_{p}\left(\mathbf{d}+e_{p}\right)=0$, then $\mathbf{b}=\mathbf{d}+e_{p}$ is a full zero point of $\Delta_{S}(\mathbf{d})$ since $\operatorname{supp}(\mathbf{b}-\mathbf{d})=\{p\}$.

Now pick an element of $S$, say $1 \in S$. By induction on size, $\Delta_{S}\left(\mathbf{d}+e_{1}\right)$ has a full zero point $\mathbf{b}$. If $b_{1}>d_{1}+1$ then $\operatorname{supp}(\mathbf{b}-\mathbf{d})=\operatorname{supp}\left(\mathbf{b}-\left(\mathbf{d}+e_{1}\right)\right)$ and $\mathbf{b}$ is a full zero 
point for $\Delta_{S}(\mathbf{d})$. So suppose $b_{1}=d_{1}+1$. If $\chi_{1}(\mathbf{b})=0$, it is a full zero point in $\Delta_{S}(\mathbf{d})$. Otherwise we have:

$$
\chi_{i}(\mathbf{b})=0 \text { for } i \in \operatorname{supp}(\mathbf{b}-\mathbf{d}) \backslash\{1\}, \quad \chi_{1}(\mathbf{b})=1 .
$$

First consider when $S$ has cardinality two, say $S=\{1,2\}$, let $\mathbf{a}=\mathbf{b}-e_{1}$ and consider the up-graph $U_{S}(\mathbf{a} ; \mathbf{d})$. Since $\chi_{1}\left(\mathbf{a}+e_{1}\right)=1$, we must in the other corner of this upgraph have $\chi_{2}\left(\mathbf{a}+e_{2}\right)=0$. Since 1 is not in the support of $\left(\mathbf{a}+e_{2}\right)-\mathbf{d}$, then $\mathbf{b}=\mathbf{a}+e_{2}$ is a full zero point.

So let $S$ have cardinality $\geq 3$ and put $S^{\prime}=S \backslash\{1\}$. We show now that each up-graph in $\Delta_{S^{\prime}}(\mathbf{d})$ has a zero corner. By induction we then have a full zero $\mathbf{b}^{\mathbf{0}}$ in $\Delta_{S^{\prime}}(\mathbf{d})$. Since 1 is not in the support of $\mathbf{b}^{\mathbf{0}}-\mathbf{d}$ this $\mathbf{b}^{\mathbf{0}}$ is also a full zero point in $\Delta_{S}(\mathbf{d})$ and we are done.

So let $U_{S^{\prime}}(\mathbf{a} ; \mathbf{d})$ be an up-graph in $\Delta_{S^{\prime}}(\mathbf{d})$. We also have the up-graph $U_{S}(\mathbf{a} ; \mathbf{d})$ in $\Delta_{S}(\mathbf{d})$. If there is some $p \geq 2$ such that $a_{p}>b_{p}$ we apply Lemma 7.12 and "pull" the point $\mathbf{b}$ to a point $\mathbf{b}^{\prime}$ in a smaller sized $\Delta_{S}\left(\mathbf{d}+e_{p}\right)$, which still contains $U_{S}(\mathbf{a} ; \mathbf{d})$. In this way we continue until we have $\mathbf{a} \mathbf{b}$ with either i) $a_{p} \leq b_{p}$ for every $p \geq 2$, or ii) the size of $\Delta_{S}(\mathbf{d})$ has become 1 . But with this size we have $\mathbf{a}=\mathbf{d}$ and so $a_{p} \leq b_{p}$ for every $p \geq 2$ in any case. We also have $a_{1}=d_{1} \leq b_{1} \leq d_{1}+1$ and recall that $|\mathbf{b}|=n$ and $|\mathbf{a}|=n-1$.

Case $b_{1}=a_{1}$. Then $\mathbf{b}=\mathbf{a}+e_{p}$ for some $p \geq 2$ and $p \in \operatorname{supp}(\mathbf{b}-\mathbf{d})$. Then

$$
\chi_{p}\left(\mathbf{a}+e_{p}\right)=\chi_{p}(\mathbf{b})=0
$$

and so $U_{S^{\prime}}(\mathbf{a} ; \mathbf{d})$ has a zero corner.

Case $b_{1}=a_{1}+1$. Then $\mathbf{b}=\mathbf{a}+e_{1}$ and $1 \in \operatorname{supp}(\mathbf{b}-\mathbf{d})$ so

$$
\chi_{1}\left(\mathbf{a}+e_{1}\right)=\chi_{1}(\mathbf{b})=1 .
$$

Since $U_{S}(\mathbf{a} ; \mathbf{d})$ has a zero corner we must have $\chi_{p}\left(\mathbf{a}+e_{p}\right)=0$ for some $p \in S^{\prime}$ and so $U_{S^{\prime}}(\mathbf{a} ; \mathbf{d})$ has a zero corner.

\section{Polarizations define Shellable simplicial Complexes}

In this section we show that the Alexander dual of any polarization of the power $(x, y, z)^{m}$ is a monomial ideal with linear quotients. This is equivalent to the polarization defining a shellable simplicial complex via the Stanley-Reisner correspondence, [17, Prop.8.2.5]. By a result of Björner [4, Thm.11.4] this immediately implies that these polarizations define simplicial balls, see Lemma 3.1 and Subsection 3.1.

An element $(a, b, c)$ in $\Delta_{3}(n-1)$ corresponds to an up-triangle in $\Delta_{3}(n)$. If $x_{\alpha} \in$ $X(a+1, b, c)$ we say that $x_{\alpha}$ (or just $\alpha$ ) is an $x$-variable belonging to the up-triangle $U(a, b, c)$. Similarly if $y_{\beta} \in Y(a, b+1, c)$ and $z_{\gamma} \in Z(a, b, c+1)$. We also say the monomial $x_{\alpha} y_{\beta} z_{\gamma}$ (or just $\alpha \beta \gamma$ ) belongs to $(a, b, c)$.

Lemma 8.1. Suppose $\alpha \beta \gamma$ belongs to the up-triangle $U(a+1, b, c)$ in $\Delta_{3}(n-1)$, see Figure 10.

a. Then either the up-triangle $U(a, b+1, c)$ or the up-triangle $U(a, b, c+1)$ has a monomial $\alpha^{\prime} \beta \gamma$ belonging to them. 


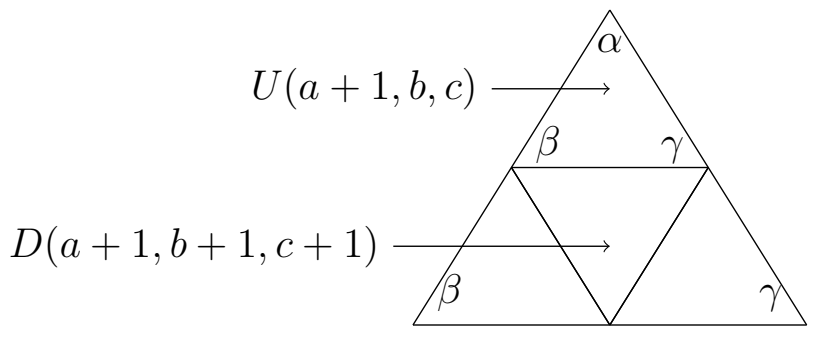

Figure 10.

b. If $\alpha$ either belongs to the up-triangle $U(a, b+1, c)$ or to $U(a, b, c+1)$, then $\alpha \beta \gamma$ will belong to one of the up-triangles $U(a, b, c+1)$ or in $U(a, b+1, c)$.

Proof. Consider the up-triangles in Figure 10. In the middle we have a down-triangle $D(a+1, b+1, c+1) \in \Delta_{3}(n)$. Note that since $Y$ is isotone, $\beta$ will be in both the up-triangles $U(a+1, b, c)$ and $U(a, b+1, c)$ and since $Z$ isotone $\gamma$ in both $U(a+1, b, c)$ and $U(a, b, c+1)$.

a. If the edge $((a+1, b+1, c+1) ; 1,2)$ is a linear syzygy edge, then also $\gamma$ belongs to $U(a, b+1, c)$, and if $((a+1, b+1, c+1) ; 1,3)$ is a linear syzygy edge then $\beta$ belongs to $U(a, b, c+1)$. Since at least one of them is a linear syzygy edge we are done.

b. If $((a, b+1, c+1) ; 2,3)$ is a linear syzygy edge, $\alpha$ is either in none or in both the two lower up-triangles. It then follows by part a that $\alpha \beta \gamma$ belongs to one of these up-triangles.

If $((a, b+1, c+1) ; 2,3)$ is not a linear syzygy edge, the two other edges are linear syzygy edges. By the argument in part a, both the lower up-triangles contains $\beta$ and $\gamma$ and so at least on of them contains $\alpha \beta \gamma$.

Let $\check{X}$ be a set of $x$-variables (with various indices) and $\check{Y}$ and $\check{Z}$ be sets of $y$ - and $z$-variables.

Lemma 8.2. Let I be an ideal generated by a subset of monomials in the product set $\check{X} \cdot \check{Y} \cdot \check{Z}$. Let $x_{\alpha} y_{\beta} z_{\gamma}$ be in $\check{X} \cdot \check{Y} \cdot \check{Z}$ but not in $I$. Then $I: x_{\alpha} y_{\beta} z_{\gamma}$ is generated by variables iff for every $x_{\alpha^{\prime}} y_{\beta^{\prime}} z_{\gamma^{\prime}} \in I$ one of the variables $x_{\alpha^{\prime}}, y_{\beta^{\prime}}$ or $z_{\gamma^{\prime}}$ is in the colon ideal.

Proof. Note that by the construction of $I$ and definition of $x_{\alpha} y_{\beta} z_{\gamma}$, none of the variables $x_{\alpha}, y_{\beta}$, or $z_{\gamma}$ can be in $I: x_{\alpha} y_{\beta} z_{\gamma}$.

That the first assertions implies the second is easy. Assume the second assertion holds. Then, if say $y_{\beta^{\prime}} z_{\gamma^{\prime}}$ is in the colon ideal, then $x_{\alpha} y_{\beta} y_{\beta^{\prime}} z_{\gamma} z_{\gamma^{\prime}}$ is in $I$. So at least some $x_{\alpha} y_{\tilde{\beta}} z_{\tilde{\gamma}}$ is in $I$, where $\tilde{\beta}=\beta^{\prime}$ or $\tilde{\gamma}=\gamma^{\prime}$. But by assumption then either $y_{\beta^{\prime}}$ or $z_{\gamma^{\prime}}$ is in the colon ideal. This implies the colon ideal is generated by variables.

We now consider the monomials $x_{\alpha} y_{\beta} z_{\gamma}$ belonging to the up-triangles $U(a, b, c) \in$ $\Delta_{3}(n-1)$ and shall provide a total order on these monomials. First consider the partial order on triples where $(a, b, c) \geq\left(a^{\prime}, b^{\prime}, c^{\prime}\right)$ if $a \geq a^{\prime}$ and take any linear extension on this to get a total order $\succeq$ on triples. 

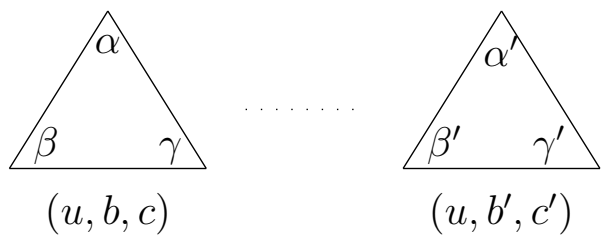

FiguRE 11.

Now for each up-triangle $U(a, b, c)$ we shall make a total order on the (degree 3 ) monomials belonging to it. For each $X(a+1, b, c)$ choose any total order of the $x$ variables. To order the variables in $Y(a, b+1, c)$ we have an ascending chain

$$
Y(a, 1, n-a-1) \subseteq Y(a, 2, n-a-2) \subseteq \cdots \subseteq Y(a, n-a, 0) .
$$

We order the variables such that each new variable popping up in the chain is less than the foregoing variables. Similarly for the variables in $Z(a, b, c+1)$ we have a chain

$$
Z(a, n-a-1,1) \subseteq Z(a, n-a-2,2) \subseteq \cdots \subseteq Z(a, 0, n-a),
$$

and we order the variables such that each new variables popping up in the chain is less than the foregoing variables. The monomials belonging to $U(a, b, c)$ correspond to

$$
X(a+1, b, c) \times Y(a, b+1, c) \times Z(a, b, c+1) .
$$

We get the partial product order on this and take a linear extension of this partial order.

We now order the monomials associated to the up-triangles in $\Delta_{3}(n)$ as follows. If $\alpha^{\prime} \beta^{\prime} \gamma^{\prime}$ occurs first in $\left(a^{\prime}, b^{\prime}, c^{\prime}\right)$ and $\alpha \beta \gamma$ occurs first in $(a, b, c)$, then

$$
\alpha^{\prime} \beta^{\prime} \gamma^{\prime}>\alpha \beta \gamma
$$

if $\left(a^{\prime}, b^{\prime}, c^{\prime}\right) \succ(a, b, c)$, or if $\left(a^{\prime}, b^{\prime}, c^{\prime}\right)=(a, b, c)$ and the order of (21) is given by the order on the monomials belonging to the up-triangle $U(a, b, c)$.

Proposition 8.3. The ideal generated by all the variables belonging to the up-triangles of $\Delta_{3}(n)$, has linear quotients given by the ordering of the monomials above.

Proof. Let $\alpha \beta \gamma$ occur for the first time in the up-triangle $(u, b, c)$ and let $I$ be the ideal generated by all the larger monomials. We shall show that $I: x_{\alpha} y_{\beta} z_{\gamma}$ is generated by variables and use Lemma $\underline{8.2}$.

1. Let $\alpha^{\prime} \beta^{\prime} \gamma^{\prime}$ be in $\left(u, b^{\prime}, c^{\prime}\right)$ where $\left(u, b^{\prime}, c^{\prime}\right) \succ(u, b, c)$. Suppose $c^{\prime} \geq c$, see Figure 11, and so $\gamma$ belongs to $\left(u, b^{\prime}, c^{\prime}\right)$, since the map $Z: \Delta_{3}(n) \rightarrow B(n)$ is isotone.

a. If $\beta \geq \beta^{\prime}$ then $\beta$ will be in $U\left(u, b^{\prime \prime}, c^{\prime \prime}\right)$ where $b^{\prime \prime} \leq b^{\prime}$ so $c^{\prime \prime} \geq c^{\prime}$. Then $\beta$ will also be in $U\left(u, b^{\prime}, c^{\prime}\right)$ and since $\gamma$ is in $U\left(u, b^{\prime}, c^{\prime}\right)$ we will have $\alpha^{\prime} \beta \gamma$ belonging to $U\left(u, b^{\prime}, c^{\prime}\right)$. If $\alpha=\alpha^{\prime}$ then $\alpha \beta \gamma$ would occur in $U\left(u, b^{\prime}, c^{\prime}\right)$ contradicting that $\alpha \beta \gamma$ first occurs in $U(u, b, c)$. So $\alpha \neq \alpha^{\prime}$ and this gives $x_{\alpha^{\prime}}$ in the colon ideal.

b. Assume now that $\beta<\beta^{\prime}$. Note that since $b^{\prime} \leq b$ we have $\beta^{\prime}$ belonging to $U(u, b, c)$. Then $\alpha \beta^{\prime} \gamma$ is already in $I$ by the ordering on the monomials belonging to $U(u, b, c)$, and hence $\beta^{\prime}$ is in the colon ideal.

2. A symmetric argument works when $\alpha^{\prime} \beta^{\prime} \gamma^{\prime}$ is in $U\left(u, b^{\prime}, c^{\prime}\right)$ and $b^{\prime} \geq b$. 

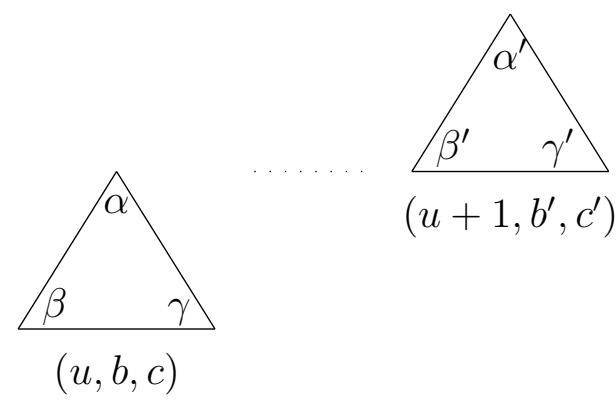

$$
\left(u+1, b^{\prime}, c^{\prime}\right)
$$

FigURE 12.

3. Assume now that $\alpha^{\prime} \beta^{\prime} \gamma^{\prime}$ belongs to the up-triangle $U\left(u+1, b^{\prime}, c^{\prime}\right)$ where the sum of these coordinates is $n-1$. Either $b^{\prime} \geq b$ or $c^{\prime} \geq c$. Suppose the latter, see Figure 12, Then $\beta^{\prime}$ belongs to the up-triangle $U(u, b, c)$ due to $Y$ being isotone.

a. If $\beta^{\prime}>\beta$ in the order given by (20), then $\alpha \beta^{\prime} \gamma>\alpha \beta \gamma$ and so the former belongs to $I$ and $\beta^{\prime}$ is in the colon ideal $I: x_{\alpha} y_{\beta} z_{\gamma}$.

b. If $\beta^{\prime}=\beta$ note that $y_{\beta}=y_{\beta^{\prime}}$ is in $Y(u, b-1, c+1)$ since $b>b^{\prime}$. By Lemma 8.1 (applied in the $y$-direction, not $x$-direction) either $\alpha \beta \gamma$ is in $U(u+1, b, c)$ or in $U(u, b-1, c+1)$. The latter must be the case since $\alpha \beta \gamma$ first occurs in $U(u, b, c)$.

c. If $\beta^{\prime}<\beta$, then since $\beta^{\prime}$ belongs to $U(u, b, c), \beta$ must belong to $U(u, b-1, c+1)$ and by Lemma $8.1 \alpha \beta \gamma$ will belong to $U(u, b-1, c+1)$.

In case b. and c. we may continue like this and push $\alpha \beta \gamma$ stepwise to the right, until we get to $(u, b-r, c+r)$ where $c+r=c^{\prime}+1$, and $(u, b-r, c+r)=\left(u, b^{\prime}, c^{\prime}+1\right)$, so $\alpha \beta \gamma$ is in both $U\left(u, b^{\prime}+1, c^{\prime}\right)$ and $U\left(u, b^{\prime}, c^{\prime}+1\right)$. Note that by $X$ being isotone $\alpha$ belongs to $\left(u+1, b^{\prime}, c^{\prime}\right)$. We show that one of $\alpha^{\prime}, \beta^{\prime}$ or $\gamma^{\prime}$ is in the colon ideal.

i. If $\beta=\beta^{\prime}$ and $\gamma=\gamma^{\prime}$ then $\alpha^{\prime} \beta \gamma$ belongs to $U\left(u+1, b^{\prime}, c^{\prime}\right)$. Since $\alpha \beta \gamma$ occurs first in $U(u, b, c)$, we cannot have $\alpha=\alpha^{\prime}$ and so $x_{\alpha^{\prime}}$ is in the colon ideal.

ii. If $\beta \neq \beta^{\prime}$ and $\gamma=\gamma^{\prime}$ then $\alpha \beta^{\prime} \gamma$ belongs to $U\left(u+1, b^{\prime}, c^{\prime}\right)$ and so $y_{\beta^{\prime}}$ is in the colon ideal.

iii. If $\beta=\beta^{\prime}$ and $\gamma \neq \gamma^{\prime}$ then $\alpha \beta \gamma^{\prime}$ belongs to $U\left(u+1, b^{\prime}, c^{\prime}\right)$ and so $z_{\gamma^{\prime}}$ is in the colon ideal.

iv. Suppose that $\beta \neq \beta^{\prime}$ and $\gamma \neq \gamma^{\prime}$. If the edge $\left(\left(u+1, b^{\prime}+1, c^{\prime}+1\right) ; 1,2\right)$ is a linear syzygy edge then $\gamma$ is in $Z\left(u+1, b^{\prime}, c^{\prime}+1\right)$ and so $\alpha \beta^{\prime} \gamma$ belongs to $U\left(u+1, b^{\prime}, c^{\prime}\right)$. If $\left(\left(u+1, b^{\prime}+1, c^{\prime}+1\right) ; 1,3\right)$ is a linear syzygy edge then $\beta \in Y\left(u+1, b^{\prime}+1, c^{\prime}\right)$ and so $\alpha \beta \gamma^{\prime}$ is belongs to $U\left(u+1, b^{\prime}, c^{\prime}\right)$.

4. Suppose then that $\alpha^{\prime} \beta^{\prime} \gamma^{\prime}$ is in $U\left(u+r, b^{\prime}, c^{\prime}\right)$ where $r \geq 2$.

a. If $b^{\prime} \leq b$ and $c^{\prime} \leq c$ (then at least one inequality is strict) then $\alpha \beta^{\prime} \gamma^{\prime}$ is in $U\left(u+r, b^{\prime}, c^{\prime}\right)$ since the map $X$ is isotone, and $\alpha$ also belongs to either $U\left(u+r-1, b^{\prime}+1, c^{\prime}\right)$ or $U\left(u+r-1, b^{\prime}, c^{\prime}+1\right)$. Hence by Lemma $8.1 \alpha \beta^{\prime} \gamma^{\prime}$ is in one of these up-triangles. We may continue until either $u+r-1=u+1$, treated in Case 3., or until $b^{\prime}>b$ or $c^{\prime}>c$. Assume $c^{\prime}>c$.

b. We then assume $\alpha^{\prime} \beta^{\prime} \gamma^{\prime}$ is in $\left(u+r, b^{\prime}, c^{\prime}\right)$ where $r \geq 2$ and $c^{\prime}>c$. Note that by $Y$ being isotone and $b^{\prime}<b, \beta^{\prime}$ will belong to $U(u, b, c)$ and to $U(u, b-1, c+1)$. 
b1. If $\beta^{\prime}>\beta$, then $\alpha \beta^{\prime} \gamma>\alpha \beta \gamma$ and so $y_{\beta^{\prime}}$ is in the colon ideal.

b2. If $\beta=\beta^{\prime}$ then $\beta$ belongs to $U\left(u+r, b^{\prime}, c^{\prime}\right)$. By $Y$ being isotone, $\beta$ belongs to $U(u, b-1, c+1)$.

b3. If $\beta^{\prime}<\beta$, then $\beta$ is in the up-triangle $U(u, b-1, c+1)$. In both cases b2 and b3, by Lemma $8.1 \alpha \beta \gamma$ is either in up-triangle $U(u+1, b-1, c)$, not possible, or in $U(u, b-1, c+1)$.

In this way we may continue going rightwards until we get to $(u, b-t, c+t)$ with $c+t=$ $c^{\prime}$. Then $\left(u, b^{\prime}+r, c^{\prime}\right)$ contains $\alpha \beta \gamma$ and so $\alpha$ is in $\left(u+r, b^{\prime}, c^{\prime}\right)$ and $\left(u+r-1, b^{\prime}+1, c^{\prime}\right)$. Then $\alpha \beta^{\prime} \gamma^{\prime}$ is in $\left(u+r, b^{\prime}, c^{\prime}\right)$ and since $\alpha \beta \gamma$ occurs first in $U(a, b, c)$ this is not equal to $\alpha \beta^{\prime} \gamma^{\prime}$. By Lemma 8.1 we may push it down to level $u+r-1$. In this way we can continue until we get $\alpha \beta^{\prime} \gamma^{\prime}$ on level $u+1$ which is treated in Case 3 .

\section{REFERENCES}

[1] Klaus Altmann, Mina Bigdeli, Jürgen Herzog, and Dancheng Lu, Algebraically rigid simplicial complexes and graphs, Journal of pure and applied algebra 220 (2016), no. 8, 2914-2935.

[2] Ekkehard Batzies and Volkmar Welker, Discrete Morse theory for cellular resolutions, Journal fur die Reine und Angewandte Mathematik 543 (2002), 147-168.

[3] Mina Bigdeli, Jürgen Herzog, Takayuki Hibi, and Antonio Macchia, Simplicial complexes of whisker type, Electron. J. Combin. 22 (2015), no. 1.

[4] A. Björner, Topological methods, Handbook of combinatorics, Vol. 1, 2, Elsevier Sci. B. V., Amsterdam, 1995, pp. 1819-1872.

[5] Anders Björner, Andreas Paffenholz, Jonas Sjöstrand, and Günter M Ziegler, Bier spheres and posets, Discrete \& Computational Geometry 34 (2005), no. 1, 71-86.

[6] Winfried Bruns and Jürgen Herzog, Cohen-Macaulay rings, Cambridge Studies in Advanced Mathematics, vol. 39, Cambridge University Press, Cambridge, 1993.

[7] Alessio D'Alì, Gunnar Fløystad, and Amin Nematbakhsh, Resolutions of co-letterplace ideals and generalizations of Bier spheres, Transactions of the American Mathematical Society 371 (2019), no. $12,8733-8753$.

[8] John A Eagon and Victor Reiner, Resolutions of Stanley-Reisner rings and Alexander duality, Journal of Pure and Applied Algebra 130 (1998), no. 3, 265-275.

[9] Giuseppe Favacchio, Elena Guardo, and Juan Migliore, On the arithmetically Cohen-Macaulay property for sets of points in multiprojective spaces, Proceedings of the American Mathematical Society 146 (2018), no. 7, 2811-2825.

[10] Gunnar Fløystad, Cellular resolutions of Cohen-Macaulay monomial ideals, Journal of Commutative Algebra 1 (2009), no. 1, 57-89.

[11] _ The colorful Helly theorem and colorful resolutions of ideals, Journal of Pure and Applied Algebra 215 (2011), no. 6, 1255-1262.

[12] _ , Poset ideals of P-partitions and generalized letterplace and determinantal ideals, Acta Mathematica Vietnamica 44 (2019), no. 1, 213-241.

[13] Gunnar Fløystad, Bjørn Møller Greve, and Jürgen Herzog, Letterplace and co-letterplace ideals of posets, Journal of Pure and Applied Algebra 221 (2017), no. 5, 1218-1241.

[14] Gunnar Fløystad and Amin Nematbakhsh, Rigid ideals by deforming quadratic letterplace ideals, Journal of Algebra $\mathbf{5 1 0}$ (2018), 413-457.

[15] Ralf Fröberg, On Stanley-Reisner rings, Topics in algebra, Part 2 (Warsaw, 1988), Banach Center Publ., vol. 26, PWN, Warsaw, 1990, pp. 57-70.

[16] Robin Hartshorne, Connectedness of the Hilbert scheme, Publications Mathématiques de l'IHÉS 29 (1966), 5-48.

[17] Jürgen Herzog and Takayuki Hibi, Monomial ideals, Monomial Ideals, Springer, 2011.

[18] Gil Kalai, Many triangulated spheres, Discrete \& Computational Geometry 3 (1988), no. 1, 1-14. 
[19] Gil Kalai and Roy Meshulam, A topological colorful Helly theorem, Advances in Mathematics 191 (2005), no. 2, 305-311.

[20] Henning Lohne, The many polarizations of powers of maximal ideals, arXiv preprint arXiv:1303.5780 (2013).

[21] Satoshi Murai, Generic initial ideals and squeezed spheres, Advances in Mathematics 214 (2007), no. $2,701-729$.

[22] (2011), no. 8, 2167-2184.

[23] Uwe Nagel and Victor Reiner, Betti numbers of monomial ideals and shifted skew shapes, The Electronic Journal of Combinatorics 16 (2009), no. 2, 3.

[24] Amin Nematbakhsh, Linear strands of edge ideals of multipartite uniform clutters, arXiv preprint arXiv:1805.11432 (2018).

[25] Ali Soleyman Jahan, Prime filtrations and Stanley decompositions of squarefree modules and Alexander duality, Manuscripta Math. 130 (2009), no. 4, 533-550.

[26] Richard P Stanley, Balanced Cohen-Macaulay complexes, Transactions of the American Mathematical Society 249 (1979), no. 1, 139-157.

[27] Richard P.Stanley, Enumerative Combinatorics, Vol.2, Cambridge Studies in Advanced Mathematics, 62 (1999).

[28] Kohji Yanagawa, Alternative polarizations of Borel fixed ideals, Nagoya Mathematical Journal 207 (2012), 79-93.

Cornell University

Email address: aka66@cornell.edu

$U R L:$ http://math. cornell.edu/ ${ }^{\sim}$ aalmousa

UNIVERSITETET I BERGEN

Email address: gunnar@mi.uib.no

UNIVERSITETET I BERGEN

Email address: lohne.henning@gmail.com 